Chapter 12

\title{
Aromatic Derivatives Based Materials for Optoelectronic Applications
}

\author{
Florin Stanculescu and Anca Stanculescu \\ Additional information is available at the end of the chapter \\ http://dx.doi.org/10.5772/51675
}

\section{Introduction}

In the last decades a high interest has been paid to the field of organic materials for electronic and optoelectronic devices as potential candidates for replacing the more expensive, energy consumer and polluting technologies involved by inorganic semiconductor devices.

One of the most important advantages of the organic materials is the possibility to modify and optimize their molecular structure using the advantages of the design at the molecular level and the versatility of the synthetic chemistry with the purpose to tune their properties and make them adequate for a well defined optic, electronic or optoelectronic application.

Organic light emitting diodes (OLED) are interesting for applications in the full-colour flat panel displays and new generation of lighting source as an alternative to incandescent bulbs and compact fluorescent lamps. The OLEDs based technology has a large area of applications from small mobile phone displays to TV and monitors because they show two important advantages compared to the competitive liquid crystals based technology: high brightness and wide viewing angle.

Since the discovery of luminescence in anthracene [1], the crucial moment in the development of the OLEDs technology was the realisation of the first organic bilayer structure able to emit light at low applied voltages [2]. After that, different organic multilayer structures have been tested to improve carrier injection, carrier transport and radiative recombination with the purpose to increase the OLED efficiency and lifetime [3-5]. The performances of the devices can be enhanced either by the selection of an adequate architecture, such as multilayer structure or by doping, by controlled impurification of the organics. 
The research was focalised on different topics such as: effect of doping of the organic semiconductor to increase the "transparency" of the energetic barrier to the injection of electron from the contact [6], influence of the trapped and interfacial charges generated in multilayer organic heterostructures on the properties of the device [7], charge tunnelling in multilayer stack and at the interface between organic and anode [8], influence of the thickness and doping of the emission layer on the properties of OLEDs [9], injection of the charge carriers from the electrodes and their migration in correlation with different types of cathodes [10-13], transport phenomena in organics $[14 ; 15]$, stacked geometry for efficient double-sided emitting OLED [16], graded mixed layer as active layer to replace heterojunction in OLEDs [17]. The awarding in 2000 of the Nobel prize for researches in the field of conducting polymers has stimulated the development of OLEDs based on polymeric materials, application emphasised years before [18], opening the way of reducing the applied voltages $(<10 \mathrm{~V})$ and increasing the brightness and lifetime.

The development of the technologies in the field of the structures for optoelectronic applications based on organic compounds is dependent of the development of fundamental and applied knowledge of all the optical and electrical processes involved, because many particularities of the organic solid state are not yet well known and understand. This is a real challenge because the number of the organic luminescent compounds is much larger than the number of inorganic compounds and it is continuously increasing. To increase the quantum efficiency, lifetime and thermal stability of these devices requires the separate optimisation of the generation, injection and transport of the charge carriers and their controlled recombination in different layers. The electrical properties of the OLED are controlled by the mobility of the charge carriers and the heights of the barriers [19], whereas the optical properties by the refractive index mismatches at the glass/air and organic/ITO interfaces that generate the trapping of a large fraction of the light by the mechanism of total internal reflection into glass and ITO [20].

Therefore the organic materials must be designed and selected in such a way to show special properties to satisfy these requirements. Organic luminescent materials can be divided considering their molecular structure and the macro scale organization. From the first point of view there are low-molecular compounds characterised by the possibility of high purification, easy vacuum deposition, high quantum yield fluorescence and large variety and high-molecular compounds (oligomers and polymers) characterised by mechanical strength, flexibility and luminescence over various spectral regions from near UV to near IR but, by small quantum yield of fluorescence. From the second point of view, macro scale organization, there are bulk crystalline organic and organic thin films and heterostructures to be used in devices' fabrication.

A special attention will be paid in this chapter to investigate the properties of bulk and thin films organic compounds showing both good optical, including luminescent, and transport properties for potential optoelectronic applications. 


\section{Bulk aromatic derivatives for optoelectronic applications}

Organic luminescent solids are attracting increasing interest in various field of application from optoelectronics to photonics. The interest in studying organic crystals is justified by the perspective to use these materials as a crystalline host matrix both for organic and inorganic guests (dopants) for developing new classes of materials combining the advantageous properties of both components host and guest. The organic matrix can assure an efficient fluorescence mechanism, can assure simple methods for processing and can contribute to electrical transport. On the other hand, the dopants could increase the charge carrier mobility and improve the emission properties and thermal stability of the organic.

Organic molecules containing electrons occupying nonlocalised molecular orbitals and strongly conjugated systems such as aromatic compounds, dyes, show important luminescence in solid state. This radiative emission involves transitions inside very well shielded systems of $\pi$-electrons. By light absorption, an electron is transferred to an antibonding $\pi$ orbital on the lowest singlet excited state with a lifetime of $10^{-6}-10^{-9} \mathrm{~s}$, from which it decays by fluorescence emission.

The perspective to tailor the specific physical properties of a molecular solid by guest particles (dopant) embedded in the crystalline organic matrix is very attractive, but not so accessible because some complications can appear both from the crystalline structure and/or dopant sites.

Special research has been devoted to the growth of organic crystals doped with rare earth metallic ions to prepare materials for luminescent and laser applications and benzil doped with $\mathrm{Cd}^{2+}$. The properties of the host/guest systems based on organic crystals depend on the crystalline perfection and chemical defects.

Growth of large and structural good organic crystals at good ratio cost/properties is very important for theoretical understanding of the phenomena taking place in organic solid state and development of new organic-organic, organic-inorganic materials for a target application. The main limitations in large-scale using of aromatic derivatives as crystalline matrix are correlated with the requirements for crystals growth, which involves identification of particularised solutions to overpass the low melting point, supercooling and low thermal conductivity of organic compounds.

Substituted aromatic molecules are a class of organic materials containing weakly coupled, strongly polarisable delocalised $\pi$ electrons. Concerning the bulk organic crystals, our interest was focalised on aromatic derivatives that contain one and two aromatic rings and substituent groups which disturb the symmetry of the $\pi$-electrons cloud, such as metadinitrobenzene (m-DNB)/ $\mathrm{C}_{6} \mathrm{H}_{4} \mathrm{~N}_{2} \mathrm{O}_{4}$ and benzil/ $\mathrm{C}_{6} \mathrm{H}_{5}-\mathrm{CO}-\mathrm{CO}-\mathrm{C}_{6} \mathrm{H}_{5}$, characterised by large transparency domain and good fluorescence emission. 


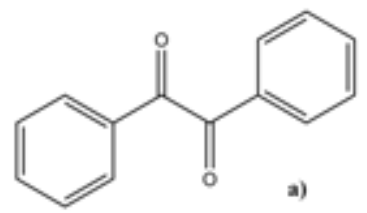

b)

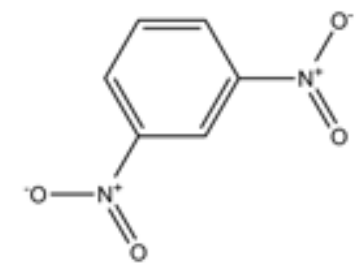

Figure 1. Benzil (a) and m-DNB (b) molecular structure [21].

Benzil with the molecular structure presented in Figure 1a is an uniaxial crystal that belongs to the space-group $D_{3}^{4}$ or $D_{3}^{6}$ and it is known as "organic quartz" being isomorphic with $\alpha$ quartz. By similarity of the microstructures developed in quartz through the diffusion of metal atoms could be of great interest to study benzil as matrix for composite materials and the effect of dopant atoms on the matrix properties.

Meta-dinitrobenzene with the molecular structure presented in Figure $1 \mathrm{~b}$ is a negative biaxial crystal that belongs to the point group symmetry $\mathrm{mm} 2$ and space group $\operatorname{Pbn} 2_{1}$.

We have developed some investigations on the effect of dopant on the emission properties (shape of the spectra, position of the peaks) of the solid-state aromatic compounds by comparison with the emission properties of the pure organic matrix. We have also evidenced the differences between the influence of the inorganic dopant (iodine, sodium, silver) and/or organic dopant (m-DNB, naphthalene) on the luminescence of bulk m-DNB and benzil samples.

\subsection{Aromatic derivatives crystal growth}

The source materials used in crystal growth must be of high purity and the purification of organic compounds is a very long process. m-DNB was purified by three methods: chemical purification, vacuum distillation and two steps directional freezing in a horizontal configuration: length of the melted zone $=2-3 \mathrm{~cm}$, average travelling speed $=2.5 \mathrm{~cm} / \mathrm{h}[22 ; 23]$.

Some factors have contributed in the selection of Bridgman-Stockbarger method in vertical configuration to grow m-DNB crystals: low melting point, low vapour pressure and no decomposition at the melting temperature. The supercooling tendency of the organic compound was counteracted in a special design system with two zones (a hot zone: $110-115^{\circ} \mathrm{C}$ generated in a furnace that assures the melting of the charge and a cold zone: $50^{\circ} \mathrm{C}$ generated by a thermostat) characterised by a steeper thermal gradient at the growth interface created by an oil bath. It is very important to correctly positioning the growth interface compared to the interface air/ oil. To allow the dissipation of the high melting heat in the organic material characterised by low thermal conductivity, the ampoule containing the crucible sealed under vacuum is moved slowly in the thermal field. The Teflon dismantle or undismantle crucible containing the organic compound powder has a special bottom configuration (a capillary tube with a diameter of 1 $\mathrm{mm}$ ) to generate the nucleation and to favour the selection of the growth direction. Details about the experimental configuration are given in Figure 2. 


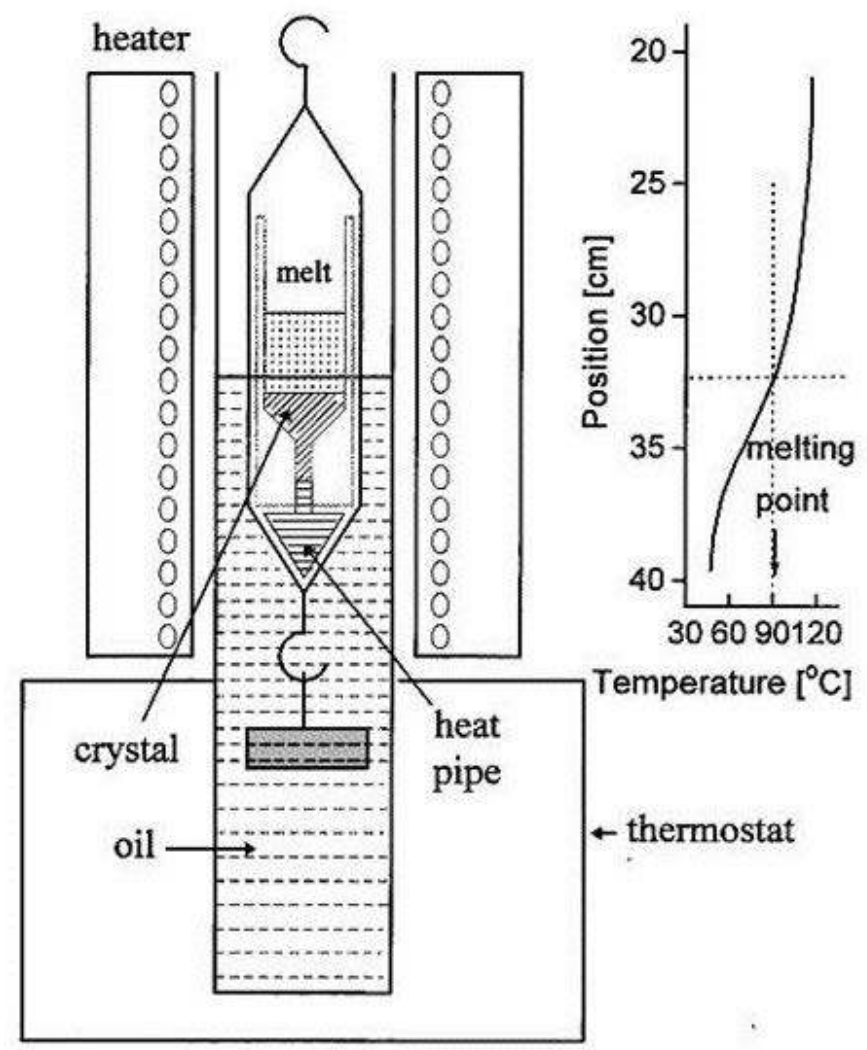

Figure 2. Experimental set-up for the growth of m-DNB crystals and the corresponding thermal profile [23].

The two parameters, thermal gradient at the melt-crystal interface of $4.5-5{ }^{\circ} \mathrm{C} / \mathrm{cm}$; $8.5-9{ }^{\circ} \mathrm{C} /$ $\mathrm{cm}$ and average moving speed of the ampoule in the furnace of $1-1.5 \mathrm{~mm} / \mathrm{h} ; 1 \mathrm{~mm} / \mathrm{h}$ are very important for the crystal growth process because are determining the shape of the solid-liquid interface and position of the growth interface with effect on the properties of the crystals.

A similar configuration, presented in Figure 3 has been used for the growth of the benzil crystals. Some differences result from the fact that benzil is characterised by a weaker adhesion to the quartz wall than m-DNB (the use of a Teflon crucible being not necessary in this case) and from the necessity to assure the control of the nucleation and solidification processes in a configuration without crucible by the use of a conical shape of the ampoule tip with a narrower zone [24]. 


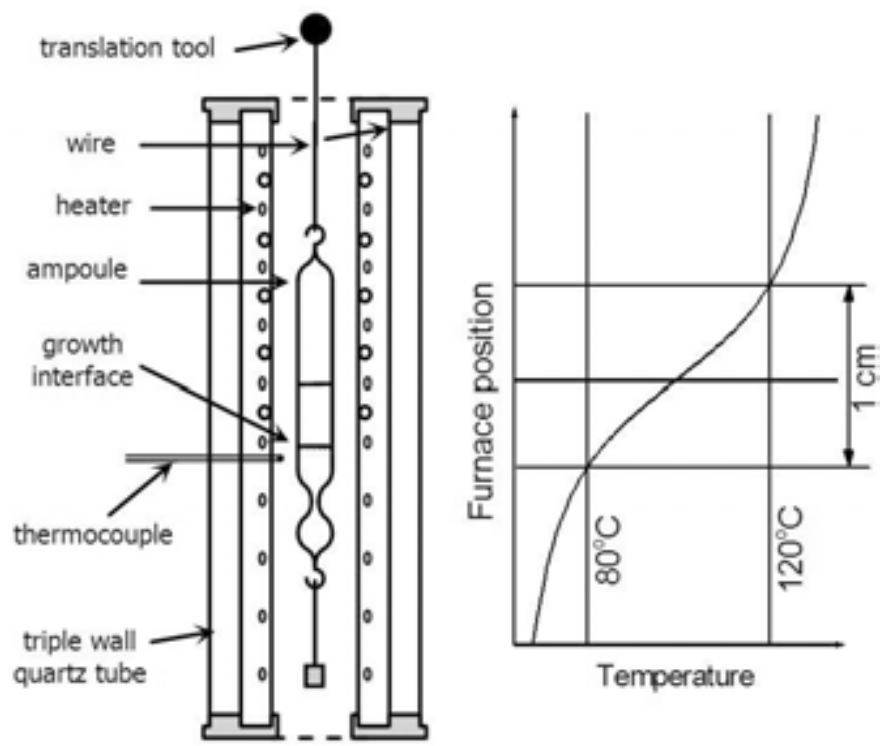

Figure 3. Experimental set-up for the growth of benzil crystals and the corresponding thermal profile [24].

A very important parameter in the process of crystals growth is the temperature, which has two counteracting actions:

1. low thermal gradients at the growth interface are necessary to prevent the generation of mechanical defects, favoured by the accumulation of tensions inside the crystal, like cracks;

2. steep gradients are necessary at the same growth interface to counteract the supercooling effect and the tendency to a facetted growth morphology [24; 25].

In general, the organic compounds are characterised by a low thermal conductivity in solid phase and high values of the solidification enthalpies that must be liberated during the crystallization process. In the system matrix/solvent can be developed many flow cells leading to non-uniform distribution of the dopant in the matrix. Constitutional supercooling characterises the doped organic melt because the freezing front rejects the particles of dopant, which can accumulate in front of the moving solid-liquid interface, the equilibrium freezing temperature of the adjacent liquid is above the actual temperature and the gradient of the equilibrium temperature is:

$$
\Delta T_{e}=\Delta C \cdot m
$$


where $\Delta \mathrm{C}=$ concentration gradient at interface; $\mathrm{m}=$ slope of the liquidus curve. $\Delta \mathrm{C} m>0$ by convention, and for molecules rejected at the interface, that decrease the melting temperature, $\mathrm{m}<0$ [26].

The problem of the growth interface stability is very important because the growth interface has effect on the quality of the obtained crystals. Our benzil/dopant system was analysed using the Mullin-Sekerka criterion [26-29], that fixes the limits of the stable growth and the conditions necessary to initiate instabilities in the growth system, and is defined by the following relation:

$$
\frac{\left(V \cdot \rho_{m} \cdot \Delta_{f} H-\left(k_{m}-k_{s}\right)-\Delta T\right)}{\left(k_{m}+k_{s}\right) \cdot(\Delta T+\Delta C \cdot m)} \geq 1
$$

where V=ampoule moving speed; $\varrho_{n}=$ melted benzil density; $\mathrm{k}_{\mathrm{m}}=$ melted benzil thermal conductivity; $\mathrm{k}_{\mathrm{s}}=$ thermal conductivity of benzil crystal; $\Delta_{\mathrm{f}} \mathrm{H}=$ solidification enthalpy; $\Delta \mathrm{T}=$ thermal gradient; $\Delta \mathrm{C}=$ concentration gradient at the growth interface; $\mathrm{m}=$ slope of the liquidus curve. For the growth of pure benzil crystals the stability condition became:

$$
\Delta C \cdot m \leq 10.0727 \cdot V-0.4382 \cdot \Delta T
$$

with $\Delta \mathrm{T}<0\left(\mathrm{~T}_{\text {final }}<\mathrm{T}_{\text {initial }}\right.$ in the solidification process $)$.

In benzil/dopant system for the given experimental conditions, the stable and unstable growth zones were delimited by the curves $\Delta C \cdot m=f(|\Delta T|)$ when $V=$ constant or by the curves $\Delta C \cdot m=f(V)$ for $\mid \Delta T \mathrm{I}=$ constant. In the first case, as can be seen in Figure 4 , at high concentration gradients $(\Delta \mathrm{C})$ the system moves through the unstable growth zone situated above the curve given by equation (3). For a given thermal gradient at the growth interface small variations in the interface moving speed have no significant influence on the area of the stable growth zone. The main consequences refer to an increase in the morphological instabilities and in crystal's homogeneity. In the second case presented in Figure 5, the area of the stable growth zone increases with the increase of the thermal gradient for a given moving speed of the growth interface, the system remaining in the stable growth zone even for high concentration gradients at the interface.

For benzil crystals $\mathrm{k}_{\mathrm{m}}>\mathrm{k}_{\mathrm{s}}$ and as consequence the interface is more stable because the term $\left(k_{m}-k_{s}\right) \cdot \Delta T /\left(k_{m}+k_{s}\right)$ in equation (2) assures a large range of values situated in the stable growth zone. 


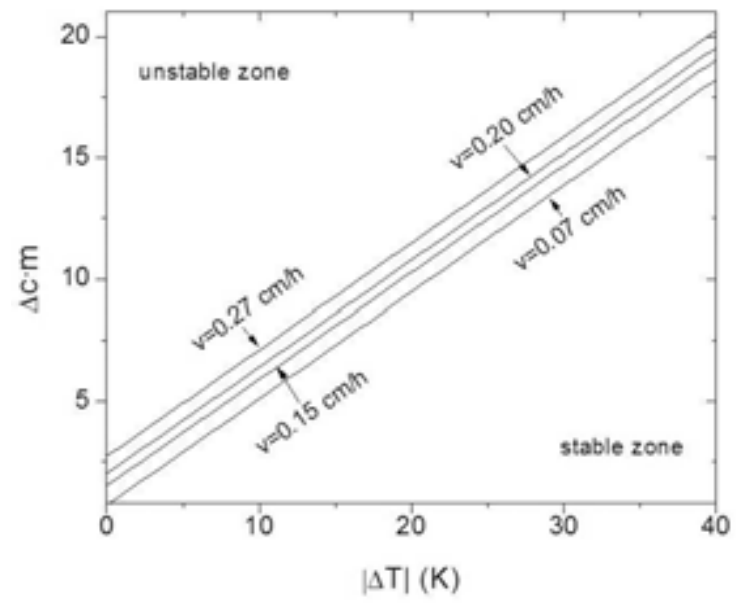

Figure 4. Stable (bellow the curves) and unstable (above the curves) growth zones for the system benzil/dopant in Bridgman-Stockbarger configuration delimited by the curves: $\Delta C \cdot m=f(|\Delta T|), V=$ constant [24].

These considerations are very important in choosing the parameters for a stable growth generating homogeneous crystals. All the studied systems based on pure and doped benzil and $\mathrm{m}$-DNB matrices are similar from the point of view of the solidus-liquidus interface stability criterion because the ratios $|\Delta T| / V$ are comparable [26; 24].

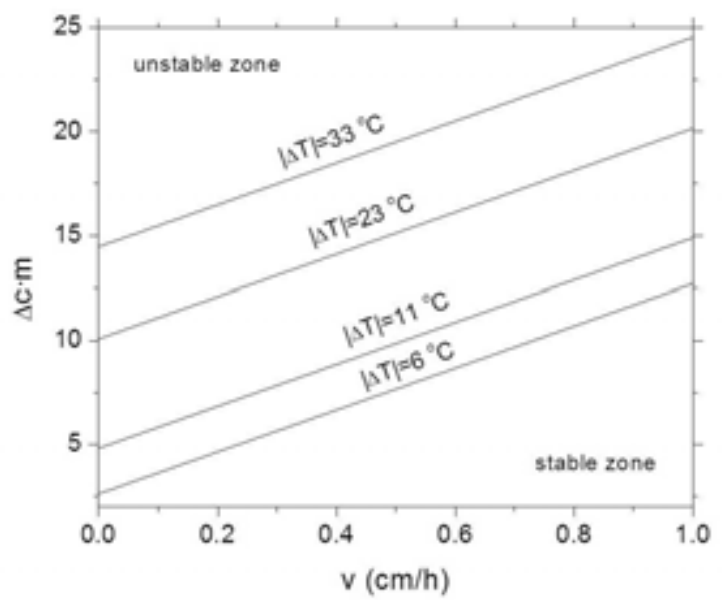

Figure 5. Stable (bellow the curves) and unstable (above the curves) growth zones for the system benzil/dopant in Bridgman-Stockbarger configuration delimited by the curves: $\Delta C \cdot m=f(V),|\Delta T|=$ constant [24]. 
The same parameters, thermal field and the interface moving speed are important in the engulfment or rejection of the dopant particles in the crystallisation front. Compositional variations and growth micrononhomogeneities (named striations) appear because of the layer situated in front of the interface, which is enriched in foreign particles by rejection. The factor which influences the incorporation of the dopant atoms/molecules in the matrix are: the shape, volume and intermolecular bonds of the dopants' molecules. A free space around 2.9 Á has been evaluated considering the molecular structure and the geometry for both benzil and $\mathrm{m}-\mathrm{DNB}$ [24]. The diameter of the dopant atoms favours the incorporation in interstitial positions and the incorporation is facilitated by the local deformation of the organic lattice characterised by weak Van der Waals forces [25; 24].

Sodium atoms could be incorporated interstitially with difficulty because the atomic diameter is greater than the diameter of the free space in benzil solid state, while silver and iodine with a diameter smaller than the free space could be easily interstitially incorporated. In the case of metals characterised by a high first ionisation enthalpy is sustained the generation of clusters, which can create difficulties in atoms incorporation (like iodine or silver).

For sodium atoms the situation is a little bit more complicated because of the high reactivity of sodium [25]. The interaction between the organic molecules and the alkali and alkaliearth metal atoms is determined by a chemical reaction leading to an organometallic complex or a charge transfer generating anion-cation pairs. The first situation is characterised by a very low probability because the hydrogen in benzil has not a very strong acid character to be directly substituted by alkali metal atoms and the crystal growth in closed sealed systems under vacuum reduces the possibility of sodium oxides formation. The charge transfer in sodium doped benzil crystals is caused by a nonbonding Van der Waals force present in all the organics and a dative bonding force corresponding to the situation in which two Na donor atoms could give each the outer 3 s electron to two oxygen atoms from the carbonyl acceptor group in benzil. Because the ionisation energy for most alkali metal atoms is low (around 4- $6 \mathrm{eV}$ ) the electron transfer from Na-guest to benzil-host is allowed without the formation of a covalent bound [25].

In the case of an organic dopant the situation is completely different and depends on the matrix. Because the organic molecules are big and can accommodate in the host lattice only substitutionally and not interstitially and must respect the condition for solubility in solid phase and the criterion for the geometrical similarity between the molecule of the dopant and matrix [30; 31]. If there are geometrical differences, the substitution is less probable and the microinclusions of dopant can generate distortion of the lattice and cracks.

The geometrical similarity is measured by the overlapping factor represented by the ratio between the unoverlapped and overlapped volume of the matrix and dopant [32]. This introduces a limitation of the doping level which can be allowed by each host/guest system. The volume of m-DNB, benzil and naphthalene molecules have been estimated supposing a spherical shape of the molecule and taking into account the length of the chemical bounds. Because the calculated unoccupied volume is much greater than the occupied volume, the possibility for m-DNB to replace benzil molecule and be included substitution- 
ally in the lattice is very small. The m-DNB molecules, which are not completely dissolved in benzil, segregate and generate microinclusions that favour the light scattering. The smaller geometrical differences between benzil and naphthalene generate weaker segregation effect.

\subsection{Optical properties of bulk aromatic derivatives}

The segregation effect of the dopant was investigated experimentally by UV-VIS measurements. The transmission of benzil doped with $\mathrm{m}$-DNB sample is lower than the transmission of benzil doped with naphthalene sample (for the same thickness $\sim 2 \mathrm{~mm}$ ) as can be seen in Figure 6, suggesting a stronger segregation of m-DNB than naphthalene in benzil matrix. The segregation of iodine in benzil matrix with effect on the homogeneity, reflected in UVVIS spectra, is stronger in the presence of another dopant (naphthalene or m-DNB) and less significant in the absence of any other organic dopant as presented in Figure 7.

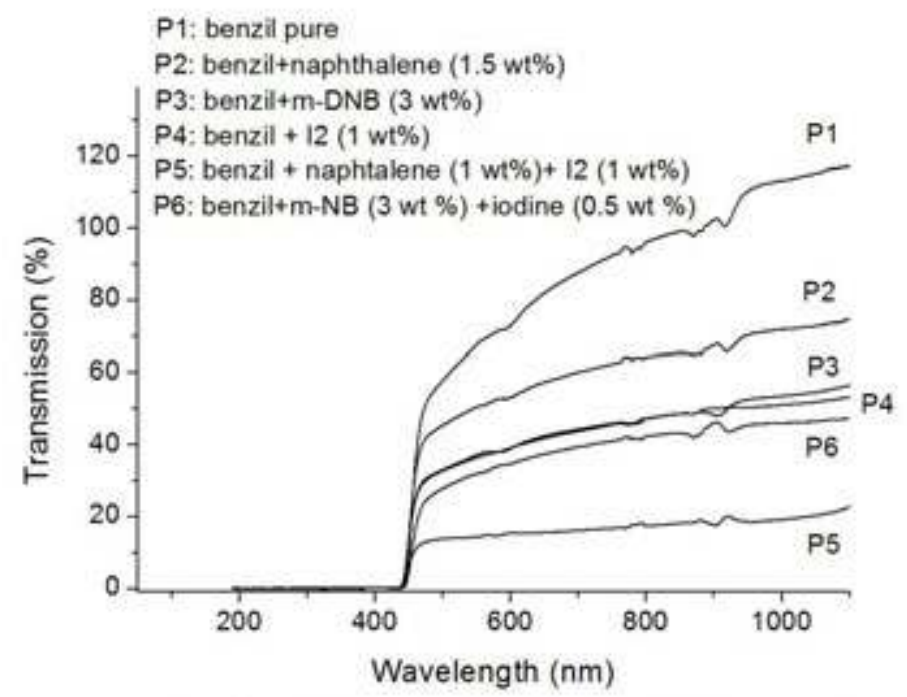

Figure 6. The effect of dopant on the UV-VIS transmission spectra of benzil matrix [24].

UV-VIS absorption spectra of pure benzil and benzil doped with Ag or $\mathrm{Na}$, presented in Figure 8, Figure 9 and Figure 10, have specific shapes characterised by a narrow peaks structure at wavelength $<450 \mathrm{~nm}$. 


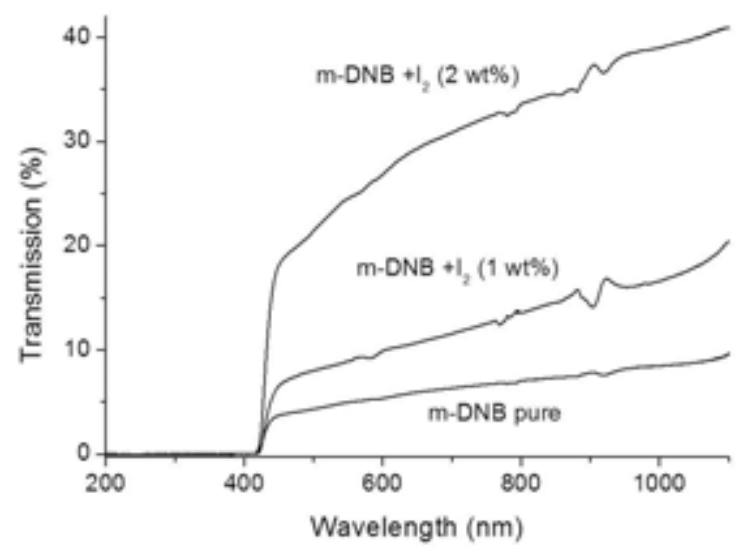

Figure 7. The effect of dopant on the UV-VIS transmission spectra of m-DNB matrix [24].

The UV-VIS spectrum of pure benzil, presented in Figure 8, preserves the pattern by doping with Ag, which is not interacting with benzil molecules. As result, the fundamental absorption edge is not affected and preserves the narrow peaks structure. The peak situated around $380 \mathrm{~nm}$ is correlated with some particularities of the benzil molecular configuration and is attributed to the absorption on the dicarbonyl groups [33; 21].

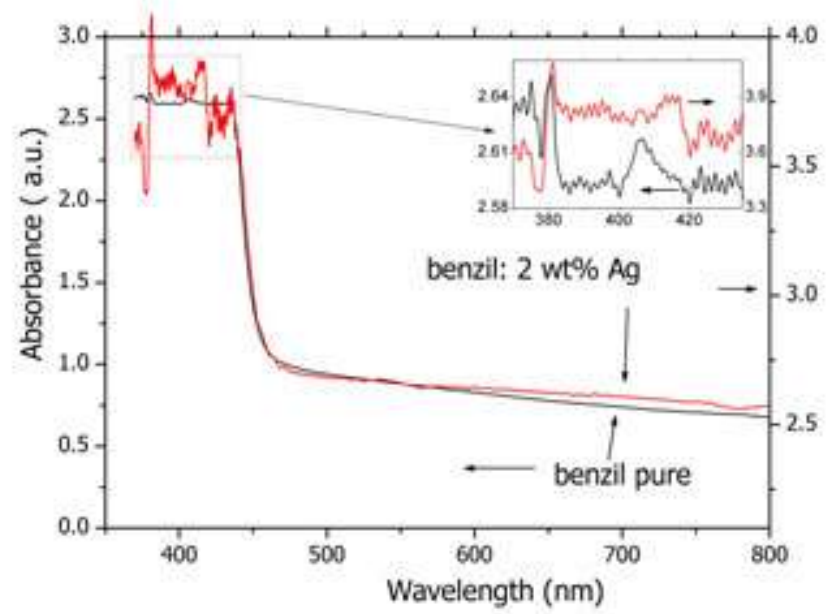

Figure 8. Comparison between the absorption spectra in bulk samples of pure benzil and benzil doped with Ag [25]. 


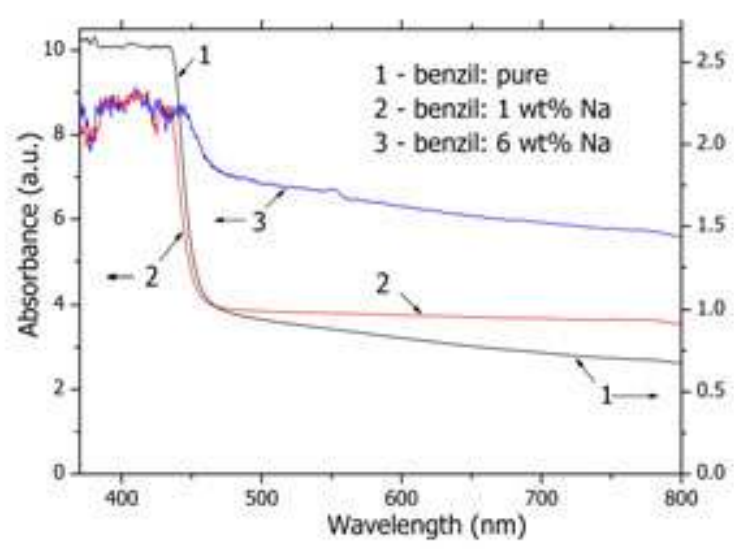

Figure 9. Comparison between the absorption spectra in bulk samples of pure benzil and benzil doped with $\mathrm{Na}$ [25].

At the contrary, doping with $\mathrm{Na}$ has introduced important changes in the shape of the fundamental absorption edge in benzil, as can be observed in Figure 9, a large structured band replacing the narrow peaks. This can be explained by the light scattering on the nonhomogeneities of the doped benzil or by changes in the forces acting between the host and guest [25].

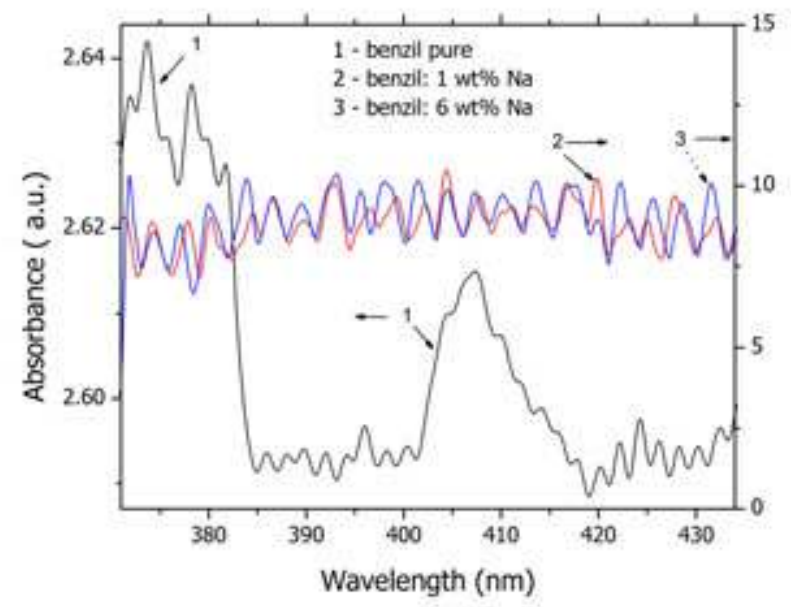

Figure 10. Absorption spectra of bulk pure and Na doped benzil matrix. Detail between $375 \mathrm{~nm}$ and $435 \mathrm{~nm}$ [25]. 
From the transmission data near the fundamental absorption edge processed using a linearpower model characterised by a formula obtained by superimposing a linear function and a power function [25]:

$$
\alpha=a+b\left(E_{g}-c\right)^{d}+m E
$$

or

$$
\alpha=a+b\left(c-\lambda_{g}\right)^{d}+m \lambda
$$

where: $\alpha=$ absorption coefficient; $\mathrm{c}=$ band gap energy, $\mathrm{E}_{\mathrm{g}}$, or edge of the fundamental absorption, $\lambda_{\mathrm{g}}$ respectively, $\mathrm{d}=$ coefficient that depends on the light absorption mechanism and (a $+\mathrm{mE})$ or $(\mathrm{a}+\mathrm{m} \lambda)$ respectively, define all the other parasitical processes, including scattering of light on the nonhomogeneities of the sample and affecting the band to band absorption mechanism.

The light absorption process is characterised by the optical band gap, which in benzil has been evaluated at $\mathrm{E}_{\mathrm{g}}=2.65 \mathrm{eV}$, emphasising the wide band gap semiconductor character of crystalline benzil. The narrowing of the optical band gap by introducing energetic levels in the band gap in pure crystal can be a consequence of physical defects or controlled doping that can generate chemical or structural defects.

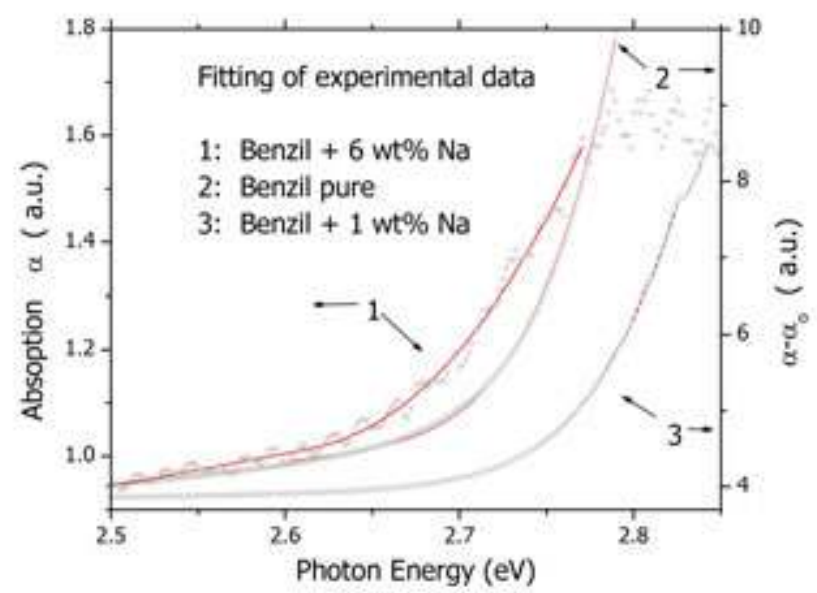

Figure 11. Fitting of the experimental data for bulk samples of pure and Na doped benzil [25]. 
As can be seen in Figure 11, the narrowing in the optical band gap is correlated with the presence of physical defects because the large radius metal atoms disturb the organic lattice and the effect of the dopant is hidden by the structural imperfections [25]. The impurities migrate and concentrate at these defects, such as grain boundaries, twins, dislocations.

For benzil doped with Ag presented in Figure 12, the situation is different and the optical absorption involves energetic levels from the band gap associated with the generation of cluster, as a consequence of the high first ionisation energy and weak reactivity of Ag.

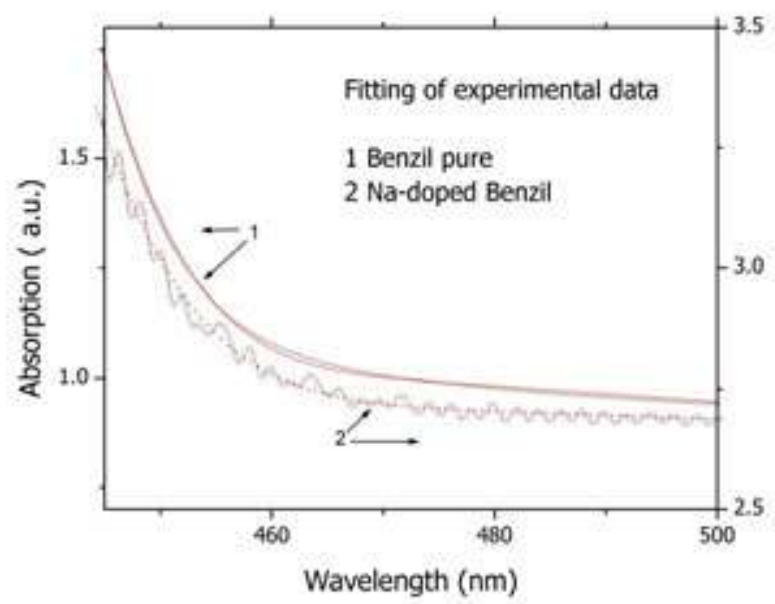

Figure 12. Fitting of the experimental data for bulk samples of pure and Ag doped benzil [25].

At excitation with $\lambda=335 \mathrm{~nm}$, the bulk sample of m-DNB shows a high, broad emission peak presented in Figure 13 [34], which correlated is with the radiative decays from the first excited energetic level, peak situated at $2.85 \mathrm{eV}$ with a shoulder at $2.95 \mathrm{eV}$ generated probably by the radiative decay from another vibrational level of the same excited energetic level.

A modification of the spectrum could be evidenced when m-DNB is doped with iodine, the position of the emission slightly moving through shorter wavelengths, the difference between the peak and shoulder being attenuated by the increase of the iodine concentration from $1 \mathrm{wt} \%$ to $2 \mathrm{wt} \%$. The blue shift of the emission peak can be associated with the migration and trapping of the exciton on the defect zones characterised by slightly higher energy compared to m-DNB without defects. Despite the strong interaction between the molecules, the energy of the level associated to the defect still remains under the energy of the exciton level.

The peak situated at $2.8 \mathrm{eV}$ evidenced both in pure and doped $\mathrm{m}$-DNB can be obtained by a radiative decay from the lowest excited triplet state to the ground state. This forbidden transition became possible by the relaxation of the selection rule in m-DNB under the effect of 
the vibrational interactions. The triplet state can be reached by a radiationless "intersystem crossing" process from the excited singlet state.

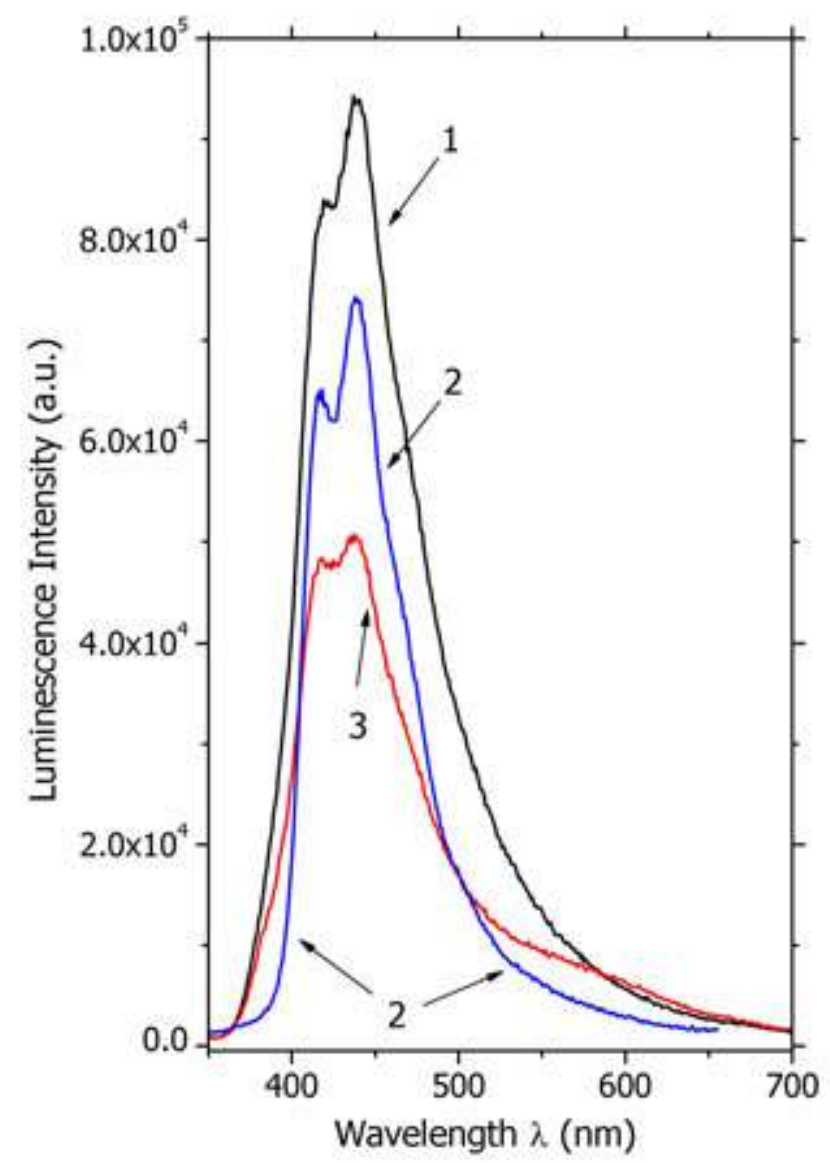

Figure 13. Luminescence spectra of m-DNB crystals: (1) pure m-DNB; (2) m-DNB doped with 1 wt \% iodine; (3) m-DNB doped with 2 wt \% iodine [34].

The m-DNB molecules contain oxygen atoms with lone electrons pairs which can be promoted to an unoccupied $\pi$ orbital and give rise to a $\left(n, \pi^{*}\right)$ singlet excite state and a triplet excited state, with an energy lower than the energy of usual $\left(\pi, \pi^{*}\right)$ state. The $\left(n, n^{*}\right)$ state in $\mathrm{m}$-DNB is the lowest excited singlet state that favours the radiationless processes as "intersystem crossing" or "internal conversion" to a lower excited energetic level. The (n, $\left.\pi^{*}\right)$ transition in m-DNB is confirmed by the small value of the "singlet-triplet" splitting evaluated from the correlation of absorption and emission data [34]. 


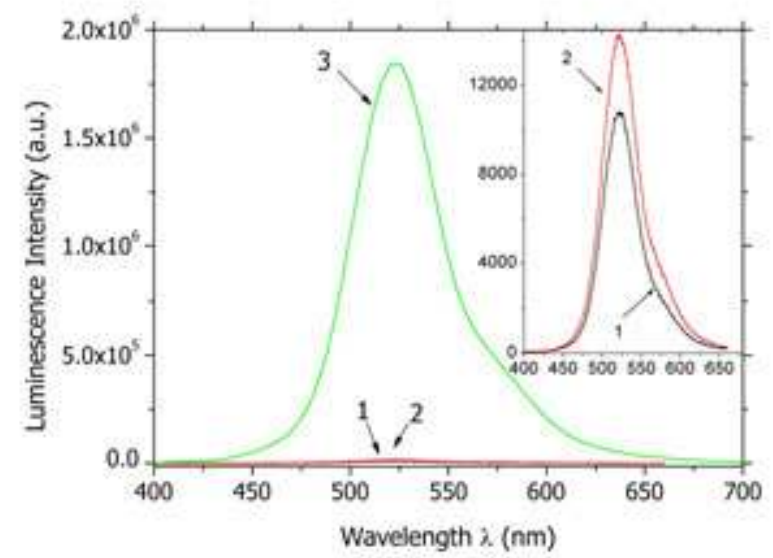

Figure 14. Luminescence spectra of benzil crystals: (1) pure benzil; (2) benzil doped with 1 wt \% iodine; (3) benzil doped with 2 wt \% iodine [34].

The luminescence spectra of pure benzil presented in Figure 14, shows an emission peak situated at $2.37 \mathrm{eV}$ generated by the lone electron pairs of oxygen atoms in carbonyl groups emitting only from planar configuration, on which are localized the emission transition involving $\left(n, \pi^{*}\right)$ states.

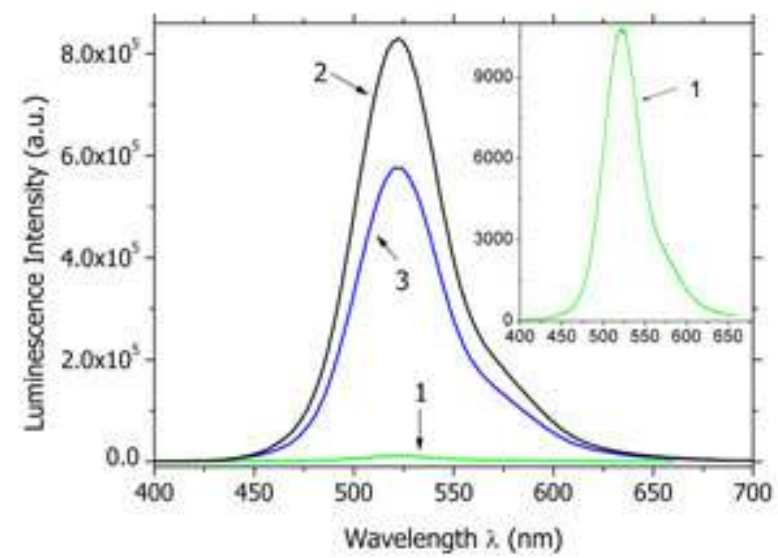

Figure 15. Luminescence spectra of benzil crystals: (1) pure benzil; (2) benzil doped with Ag (2.4 wt \%); (3) benzil doped with $\mathrm{Na}(1 \mathrm{wt} \%)$ [34]. 
The doping with other metallic impurities such as silver (2.4 wt\%) or $\mathrm{Na}$ ( $1 \mathrm{wt} \%$ ) has not modified the sharp peak situated at $2.37 \mathrm{eV}$, which is present in pure benzil, as it is emphasised in Figure 15, and this peak could not be correlated with an exciton trapping mechanism [34]. The peak at $2.37 \mathrm{eV}$ could be generated by the radiative decay from the excited triplet state $\left(\mathrm{T}_{1}\right)$ to the ground state $\left(\mathrm{S}_{0}\right)$, which is a transition forbidden for separated molecules becoming allowed through the vibrational interactions when the molecules are coupled in the solid state, in a crystalline lattice $[23 ; 35 ; 36]$.

In Figure 16 is presented the spectrum of benzil doped with m-DNB. The benzene derivative, $\mathrm{m}-\mathrm{DNB}$, is active itself and has a direct action on the benzil matrix. The peak assigned to $\mathrm{m}$-DNB is situated at $2.97 \mathrm{eV}$ in the high-energy range of the emission spectrum. The preferential excitation of $\mathrm{m}$-DNB molecule can be explained by the lower position of the excited singlet state $(2.9 \mathrm{eV})$ in $\mathrm{m}$-DNB compared to benzil $(3.25 \mathrm{eV})$. At the contrary, naphthalene has not any significant influence on the emission spectrum of benzil presented in Figure 17, because the first singlet excited state in naphthalene is situated at $\sim 3.84 \mathrm{eV}$ and the triplet state at $\sim 2.64 \mathrm{eV}$ higher than the corresponding energetic levels in benzil ( $3.25 \mathrm{eV}$ and $2.3 \mathrm{eV}$ respectively). Therefore the peak observed in the emission spectrum of naphthalene doped benzil is obtained by the radiative deexcitation of benzil matrix.

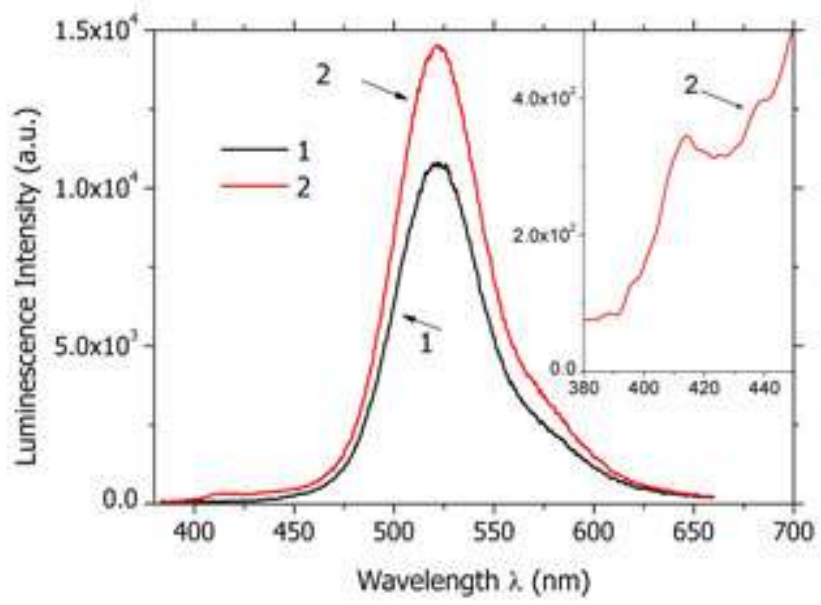

Figure 16. Luminescence spectra of benzil crystals: (1) pure benzil; (2) m-DNB doped benzil (3 wt \%) [34].

As can be seen from Figure 17, the simultaneous doping with naphthalene and iodine has no significant effect on shape of the emission spectrum of benzil. 


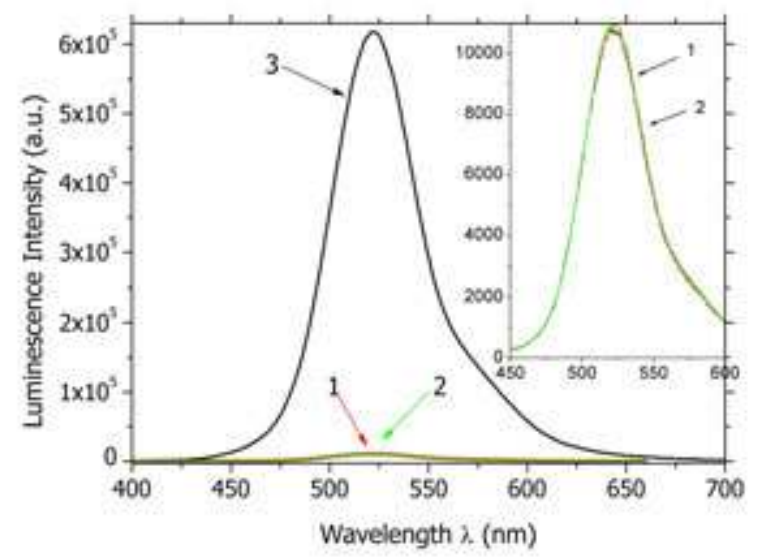

Figure 17. Luminescence spectra of benzil crystals: (1) pure benzil; (2) benzil doped with naphthalene (1.5 wt \%); (3) benzil doped with naphthalene (1 wt \%) and iodine (1 wt \%) [34].

\section{Aromatic derivatives thin films for optoelectronic applications}

The major problems for large-scale application of crystalline matrices from these aromatic derivatives materials are associated with the difficulties to grow (low melting point, supercooling, and low thermal conductivity), process (weak mechanical properties determined by weak bonding forces between molecules) and doping organic crystals to assure an homogeneous distribution of the guest atoms.

Crystalline organic films are preferred in a variety of applications because of the complexity of the processes involved and long time necessary to grow bulk organic crystals. The use of thin films is more promising because represents an optimum between the cost of manufacturing and properties of interest for special and oriented applications.

Investigation of the properties of organic thin films is a very important aim because these films are components of the organic heterostructures as fundamental elements of any organic devices. It is also necessary to investigate the properties of heterostructures because the junction between two different semiconductors (organic/organic; organic/inorganic) or between a metal and a semiconductor (metal/organic) is the key building block of any modern electronic, photovoltaic and optoelectronic devices. Heterojunction technology has known a continuous development from the first heterojunction transistor, realized by Bardeen in 1948 at Bell Laboratory [37], to p-n junction transistor, realized by Schockley in 1949 [38], and to nowadays devices based on multilayer heterostructures.

The organic compounds which have been investigated as components of organic compound based heterostructures are: 
a. 3,4,9,10-perylenetetracarboxylic dianhydride (PTCDA);

b. Zinc phthalocyanine $(\mathrm{ZnPc})$;

c. tris(8-hydroxyquinoline) aluminium (Alq3) and

d. 5, 10, 15,20-yetra(4-pyrydil)21H, 23H-porphyne (TPyP).

PTCDA is known as having $\mathrm{p}$ type conduction while Alq3 and TPyP are characterized by $\mathrm{n}$ type conduction. They molecular structure is given in Figure 18.

$\mathrm{ZnPc}$ is an electron donor forming highly ordered layer, with a broad transmission window in visible region of the spectrum [39].

In PTCDA, an electron acceptor, the interaction between the $\pi$-electrons systems is favored by the planar molecule and the perpendicular stacks of molecular planes [40], which determine a quasi-one-dimensional molecular crystal structure [41].

Alq3 shows different stereoisomers (median, facial) determined by the mutual orientation of the ligands of hydroxyquinoline, which show different symmetries and as consequence different properties $[42 ; 43]$.
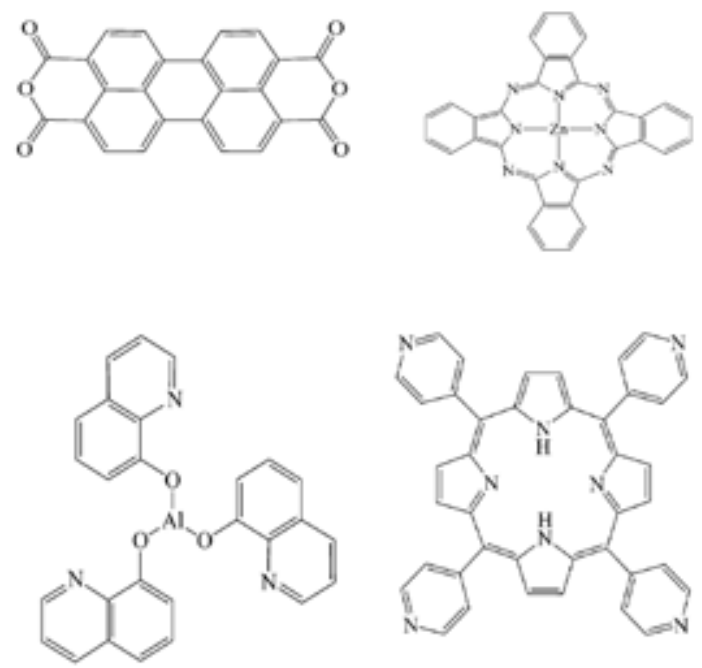

Figure 18. PTCDA (a), ZnPc (b), Alq3 (c) and TPyP (d) molecular structure.

TPyP is a non-metallic porphyrin with an increased electron affinity obtained by the substitution of phenyl group by pyridyl group determining the $n$ type conduction. The basic structure of porphyrin consists in four pyrrolic entities linked by four unsaturated methane bridges with a skeleton showing an extended $\pi$-electrons system assuring a large spectral range for light absorption [44]. 


\subsection{Preparation of the aromatic derivatives thin films}

\subsubsection{Aromatic derivatives thin films preparation by directional solidification process}

It is not very easy to obtain organic thin films because of the same difficulties which affect the preparation of organic crystals and the quality of the organic layer is strongly influenced by the method, which have been selected to grow the film. For example there is a high concentration of structural defects in the benzil thin films which have been grown by a rapid directional solidification process, characterised by a non rigourous control of the thermal regime compared to the crystal growth process and these defects have caused the red shift of the emission peak. It is very difficult to grow, by vacuum evaporation, thin films of organic compounds characterized by a melting point $\mathrm{T}_{\mathrm{m}}<100{ }^{\circ} \mathrm{C}$ (including benzil and m-DNB) because the heating of the substrate during the evaporation process can favor a strong desorption of the organic molecules from the substrate. By the directional solidification process could be prepared organic thin films between two substrates, like quartz or glass substrates, during a rapid thermal solidification characterized by a temperature gradient for solidification, $\Delta \mathrm{T}>50^{\circ} \mathrm{C}$, necessary to counteract the supercooling phenomenon [45]. Crystalline fragments from organic ingots of pure and doped m-DNB $\left(\mathrm{T}_{\mathrm{m}}=89.9^{\circ} \mathrm{C}\right)$ or benzil $\left(\mathrm{T}_{\mathrm{m}}=95{ }^{\circ} \mathrm{C}\right)$, grown by Bridgman-Stockbarger method presented in paragraph 2.1, have been melted between the substrates by the hot plate technique and after that rapidly frozen by the cold plate technique obtaining films with a columnar structure with large dendrites branches in the plane of the film determined by the low thermal conductivity and anisotropy of these organic compounds. The thickness of the films has been evaluated (using the density in solid state) from geometrical considerations presuming that the total volume of the substance didn't change during the melting-solidification cycles.

\subsubsection{Aromatic derivatives thin films and heterostructures preparation by vacuum evaporation}

Thin films of PTCDA, Alq3 and TPyP have been prepared by vacuum evaporation and deposition on different substrates (glass/ITO, quartz, Si), which have been cleaned in acetone (glass/ITO, quartz) and with acetone, hydrofluoric acid and distillate water (Si).

Stable, homogeneous organic films, with good adhesion to the substrates have been prepared, by the evaporation of the organic powder contained in the quartz crucible heated by a self-sustaining kanthal winding, in an Alcatel system with turbo molecular pump [40;46]. During the deposition with a duration between 10-15 min, the temperature was measured by a thermocouple situated at the bottom of the crucible and varied between $220-240{ }^{\circ} \mathrm{C}$ for PTCDA [40], $150-160{ }^{\circ} \mathrm{C}$ for Alq3 [40] and $175-185^{\circ} \mathrm{C}$ for TPyP [46].

\subsubsection{Aromatic derivatives thin films an heterostructures preparation by MAPLE}

A special type of Pulsed laser Deposition (PLD) technique, Matrix Assisted Pulsed Laser Evaporation (MAPLE), has been used for the deposition of small molecule organic films (PTCDA, ZnPc, Alq3). This technique involves the ablation of a target formed by the frozen solution of the organic compound in a high molecular weight and strong laser wavelength 
absorbing solvent, like dimethylsulphoxide (DMSO) or chloroform. Deposition was realized with a $\mathrm{KrF}^{*}$ laser, Coherent ComplexPro 205 characterised by $\lambda=248 \mathrm{~nm}, \tau_{\mathrm{FWHM}} \sim 25 \mathrm{~ns}$, repetition rate $=10 \mathrm{~Hz}$ [47]. The incident laser energy absorbed by the solvent molecules is converted into thermal energy determining the heating and simultaneous evaporation of the two components. The solvent molecules are pumped away by the vacuum pump that maintains a pressure of $10^{-2}-10^{-1}$ mbar in the deposition chamber, while the less volatile molecules of the organic compound deposit on the substrate maintained at room temperature. The low value fluence varied between $160 \mathrm{~mJ} / \mathrm{cm}^{2}$ and $430 \mathrm{~mJ} / \mathrm{cm}^{2}$ to avoid the deterioration of the organic molecule and the number of pulses between 10000 and 120000, with effect on the films' thickness ( $40 \mathrm{~nm}-150 \mathrm{~nm}$ ), which has been evaluated by ellipsometry.

\subsection{Optical properties of aromatic derivatives thin films}

The absorption spectrum of m-DNB, presented in Figure 20, which is similar to a classical semiconductor, could be correlated with the strong interactions between the polar molecules and with the partial superposition of the $\pi$-electrons clouds from neighbour molecules generating narrower valence and conduction bands. This spectrum is different from that of benzil presented in Figure 19, which is characterized by a two edges of the fundamental absorption, with a subband light absorption peak situated at $380 \mathrm{~nm}$, attributed to absorption by dicarbonyl groups, strongly interacting in the solid state and producing the split of the energetic level $\left(n, \pi^{*}\right)$.

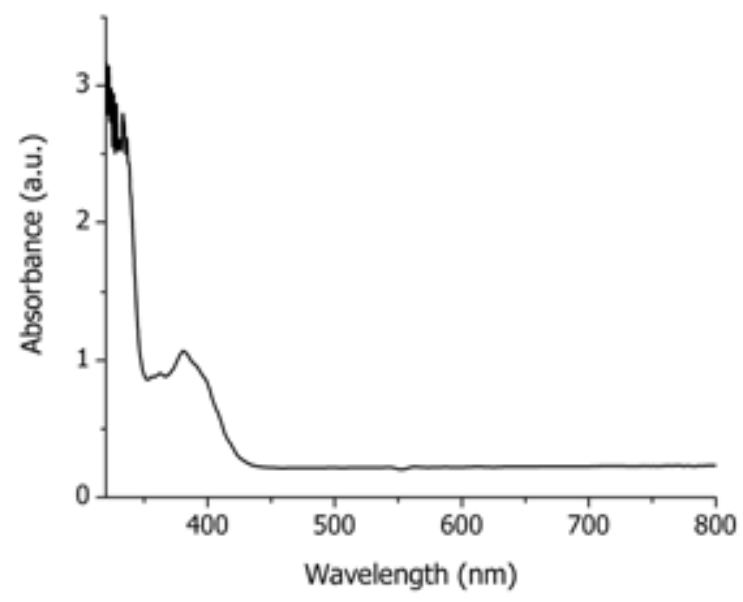

Figure 19. Absorption spectra of benzil film grown between two quartz plates [45]. 


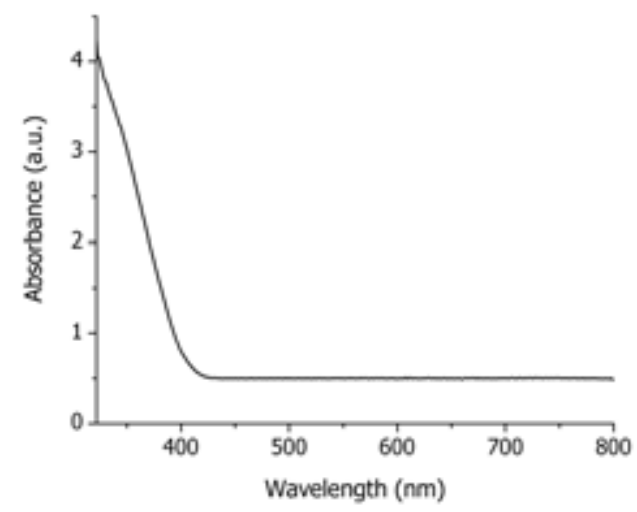

Figure 20. Absorption spectra of m-DNB film grown between two quartz plates [45].

From Figure 21 it can be emphasized that the shape of the fundamental absorption edge is not affected by the presence of impurities. No important changes have been evidenced at the absorption edge characterized by a lower energetic threshold. But the absorption at the edge characterized by the higher energetic threshold is attenuated in benzil doped with m-DNB or sodium compare to pure benzil because of the light scattering process on the nonhomogeneities of the films. This effect is stronger in benzil doped with $\mathrm{Na}$ because it is not completely dissolved, segregates and generates microinclusions as a distinct phase.

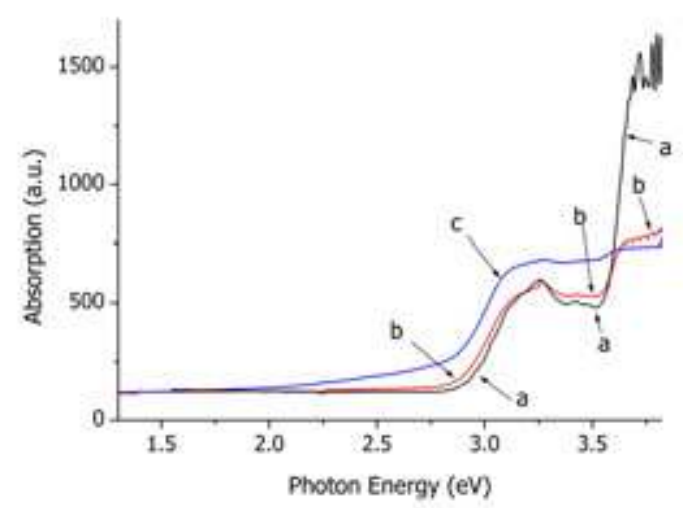

Figure 21. Comparative absorption spectra of: pure benzil (a); benzil doped with m-DNB (3 wt \%) (b); benzil doped with $\mathrm{Na}(6 \mathrm{wt} \%)(\mathrm{c})$ : grown between two quartz plates [45]. 
The effect of the impurities on the shape and position of the absorption peak in benzil situated at $3.25 \mathrm{eV}$ and assigned to dicarbonyl group absorption is not important and no other absorption peaks have been evidenced in the longer wavelength range to sustain the trapping of the excitation energy by theses impurities. Therefore we can conclude that the energetic levels of these impurities are not significantly lower than the lowest energetic level which characterizes the crystalline assembly [21].

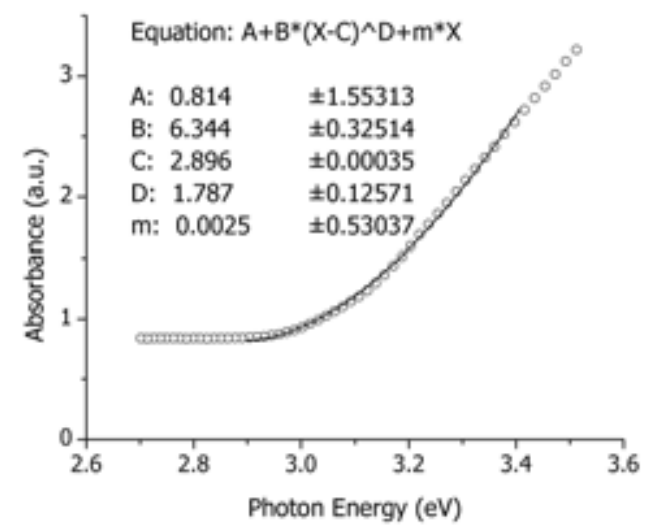

Figure 22. Fitting of the experimental data for pure m-DNB film [45].

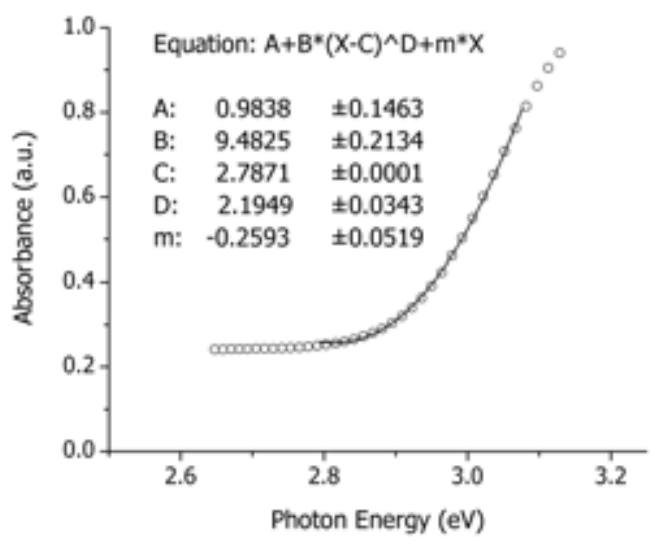

Figure 23. Fitting of the experimental data for pure benzil film in the range $2.6 \mathrm{eV}-3.1 \mathrm{eV}$ [45]. 


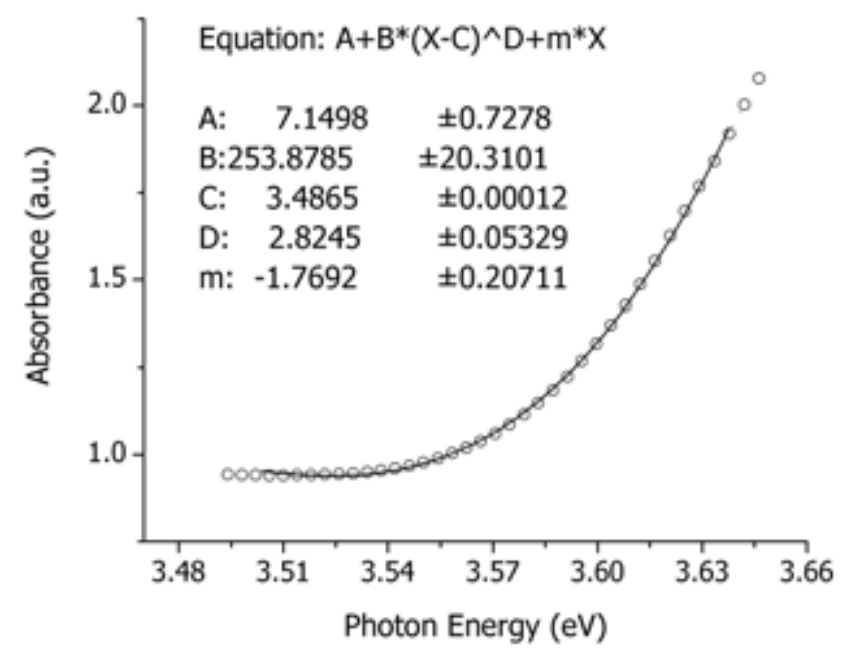

Figure 24. Fitting of the experimental data for pure benzil film in the range $3.49 \mathrm{eV}-3.65 \mathrm{eV}$ [45].

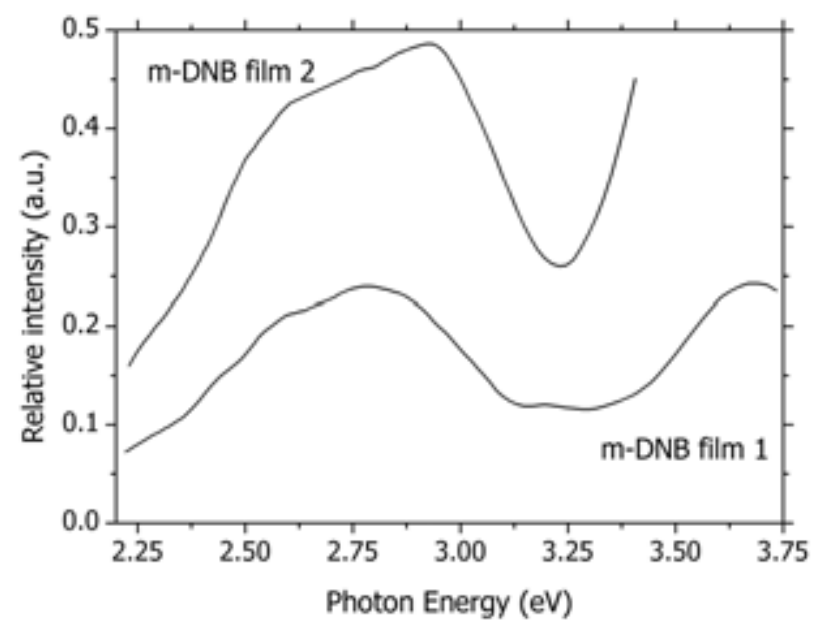

Figure 25. Emission spectra of pure m-DNB films of different thickness for $\lambda_{\text {excitation }}=300 \mathrm{~nm}$ [21]

The band gap energy has been evaluated from the experimental data fitting, near the fundamental absorption edge, shown in Figure 22 for m-DNB and, Figure 23 and Figure 24 for 
benzil, using the formula (4) and we have obtained $E_{g}=2.90 \mathrm{eV}$ for $\mathrm{m}-\mathrm{DNB}$ and $\mathrm{E}_{\mathrm{g} 1}=2.79 \mathrm{eV}$ and $\mathrm{E}_{\mathrm{g} 2}=3.54 \mathrm{eV}$ for the two absorption edges in benzil.

Supplementary information about the optical properties of theses aromatic derivatives in solid state have been obtained from the luminescence measurements. At excitation with $\lambda=300 \mathrm{~nm}$, the emission spectra of pure m-DNB present a peak situated at $2.92 \mathrm{eV}$ with a shoulder at $2.63 \mathrm{eV}$, as it is shown in Figure 25. The apparent red shift of the emission peak situated at $2.92 \mathrm{eV}$ to $2.78 \mathrm{eV}$ in thicker film is due to a self-absorption process of the emitted radiation and not to a recombination on the energetic levels associated with physical defects or impurities.

Luminescence spectra of benzil films presented in Figure 26 show a peak at $2.30 \mathrm{eV}$ attributed also to the radiative decay from the excited triplet state $\left(T_{1}\right)$ to the ground state $\left(S_{0}\right)$, transition possible because of the vibrational interactions as was mentioned for bulk samples in paragraph 2.2.

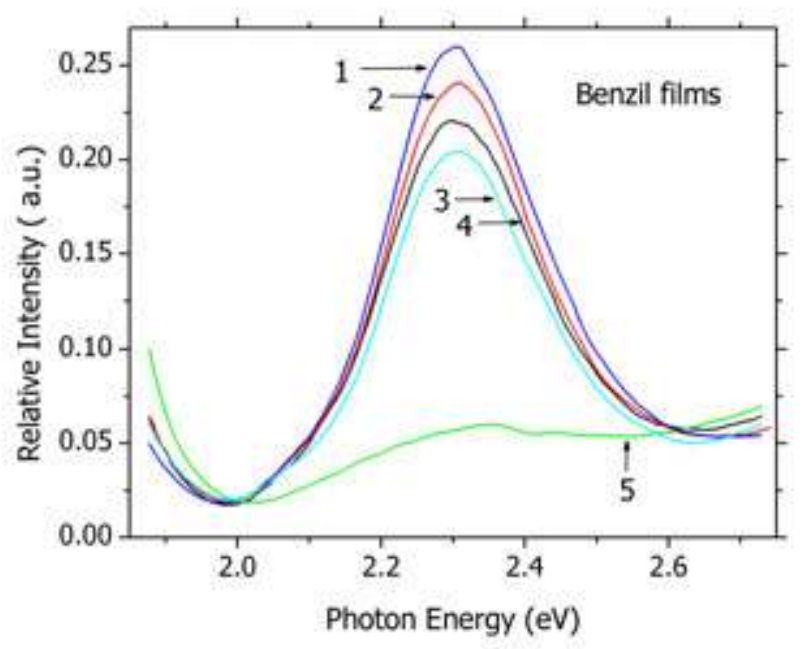

Figure 26. Emission spectra of pure and doped benzil films: (1) benzil doped with Na (1 wt \%); (2) benzil doped with m-DNB (3 wt \%); (3) benzil doped with Ag (2.4 wt \%); (4) benzil pure; (5) benzil doped with Na (6 wt \%) [21].

The excited molecules of benzil are more sensitive to a radiationless process and the conversion from the first singlet excited state to the lowest triplet excited state becomes possible by "intersystem crossing", transition between states with different multiplicity, very efficient in systems containing carbonyl groups, like benzil. This radiationless process is followed by a radiative decay through phosphorescence from $\left(T_{1}\right)$ to $\left(S_{0}\right)$. The intersystem crossing appears in benzil as a transition between $\left(n, \pi^{*}\right)$ states of singlet and triplet levels rather than between $\left(\pi, \pi^{*}\right)$ states. Both absorption and emission transitions involve states localized on carbonyl groups, which emit only from planar configuration. 
The behaviour of benzil molecule depends on the flexible conformation [48] because the torsion angle around the central bond $\mathrm{C}-\mathrm{C}$ can change and the geometry of the molecule can change after excitation with light. In the ground state the benzil molecule has a skew configuration and can twist around the carbonyl-carbonyl bound with little interaction between the two benzyl halves of the molecule. This interaction becomes strong in excited molecule, which rearranges in a new configuration with a trans-planar dicarbonyl system characterized by a redistribution of the energy followed by the process of „intersystem crossing". By phosphorescent emission the system passes from a trans-planar configuration to the ground state with also a trans-planar configuration considering the Frank-Condon principle [49]. In the next step the molecule relax from the emissive transplanar configuration to a skew configuration and the differences in the emission spectra of benzil can be determined by changes in the molecular conformation of the ground state [50]. For most of the dopants we have not remarked any modification in the shape and position of the emission peak that sustains no modification in the molecular conformation of the $\left(n, \pi^{*}\right)$ state with effect on the angle between the carbonyl groups. The only changes, a slightly blue shift and significant attenuation in intensity, have been remarked in Figure 26 for benzil highly doped with Na (6 wt \%). A possible charge transfer from $\mathrm{Na}$ atoms to oxygen atoms in carbonyl groups can generate conformational changes, and the shift could be explained by the decrease in the dihedral angle between the two carbonyl groups [21]. The only impurity active in emission is sodium at high concentration because of the conformational changes in the emitting triplet state, while $\mathrm{m}-\mathrm{DNB}, \mathrm{Ag}, \mathrm{Na}$ are not active in absorption.

In Figure 27 and Figure 28, we have emphasized the band structure of the absorption spectra in PTCDA and Alq3 with peaks situated at $358 \mathrm{~nm}, 374 \mathrm{~nm}, 475 \mathrm{~nm}, 552 \mathrm{~nm}$ and $232 \mathrm{~nm}$, $261 \mathrm{~nm}, 380 \mathrm{~nm}$ respectively. This confirms the presence of median isomer in Alq3 film. The position of the two important peaks situated at $2.25 \mathrm{eV}$ and $2.61 \mathrm{eV}$ remain unchanged in the absorption spectrum of PTCDA deposited on glass covered by ITO while the absorption spectrum of the heterostructure with double organic layer, glass/ITO/PTCDA/Alq3, preserves the pattern of the absorption spectrum of PTCDA between $400 \mathrm{~nm}$ and $600 \mathrm{~nm}$, which is very important in the stage of the charge carrier's generation.

In Figure 29 have been evidenced the presence of $\pi-\pi^{*}$ absorption bands characteristic for free-base ethio type porphyrin. These bands are associated with $\pi-\pi^{*}$ transition between bonding and anti-bonding molecular orbitals. Other bands which have been also evidenced are: an intense Soret band (B) with a peak centred at $430 \mathrm{~nm}$ and $4 \mathrm{Q}$ bands situated at 520 $\mathrm{nm}, 555 \mathrm{~nm}, 590 \mathrm{~nm}$ and $645 \mathrm{~nm}$. 


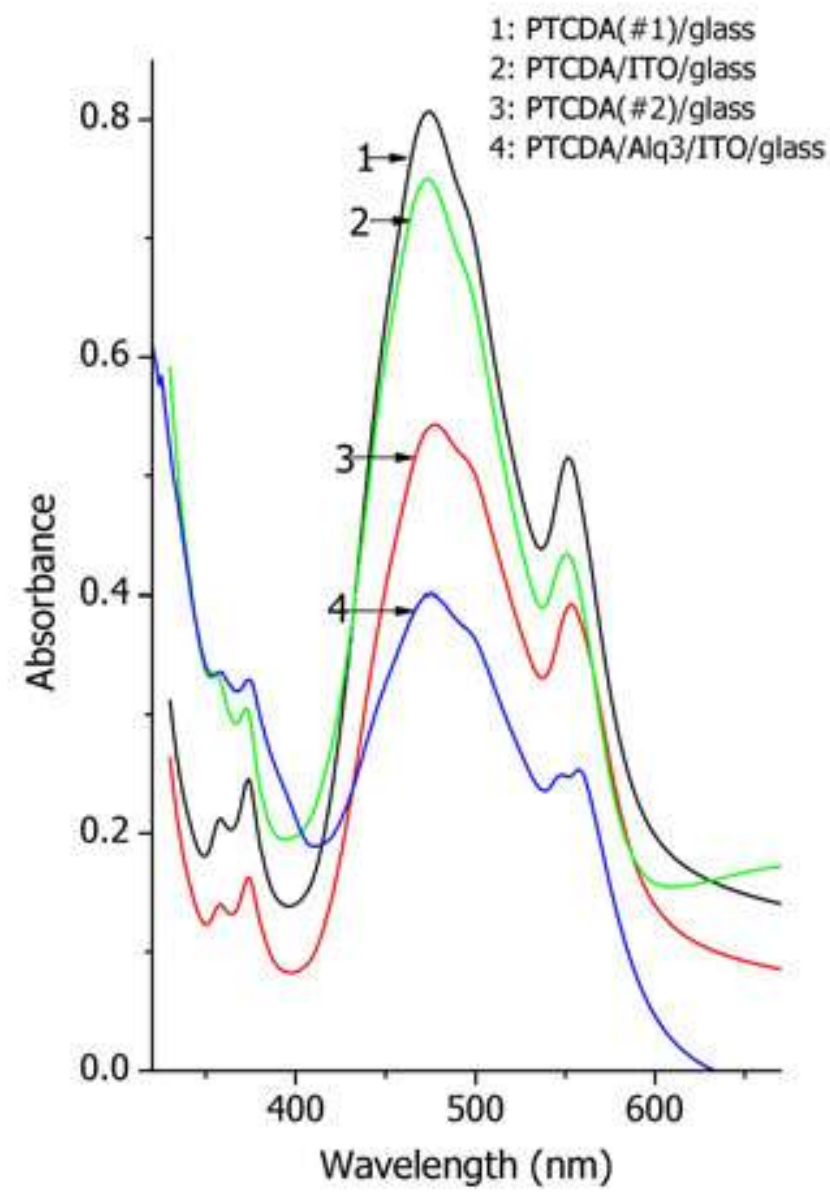

Figure 27. Absorption spectra of PTCDA thin films deposited by vacuum evaporation [40]. 


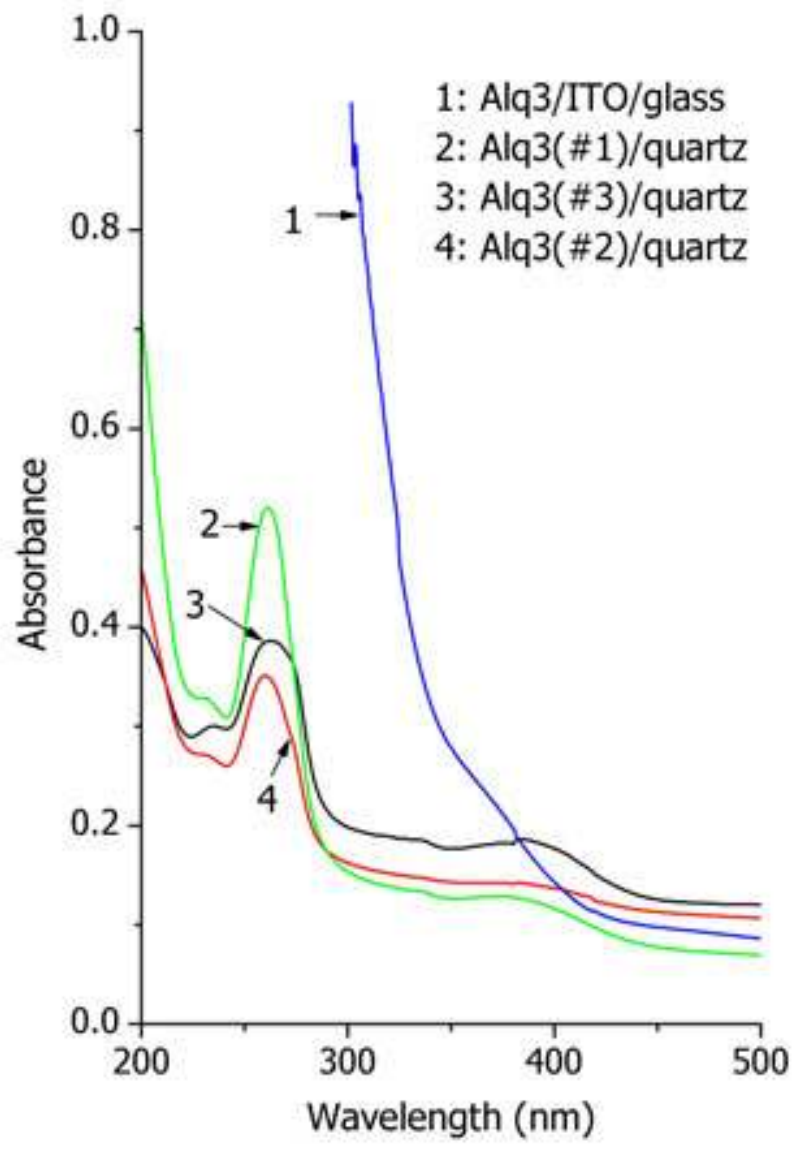

Figure 28. Absorption spectra of Alq3 thin films deposited by vacuum evaporation [40]. 


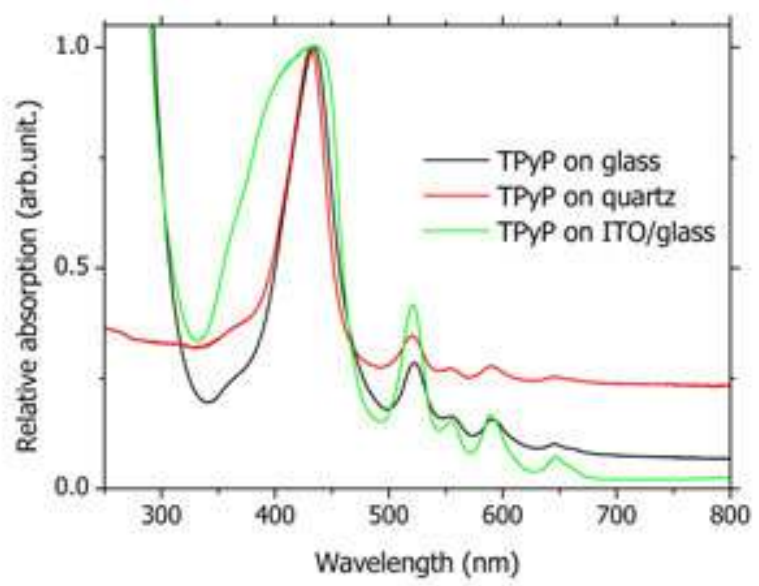

Figure 29. Absorption spectra of TPyP thin films deposited by vacuum evaporation [46].

The shape of the absorption spectra of TPyP thin films deposited on different substrates is preserved at $\lambda>430 \mathrm{~nm}$. The slight red shift of the Soret band can be determined by the order induced by the interaction between the molecules in solid state influenced by the interaction with the substrate [51]. A possible bonding mechanism can be based on the pyridylsurface interaction mediating the deformation of the molecule after adsorption on the substrate's surface. Subsequent packing of the molecules can be determined by the non-covalent interactions mediated by the terminal pyridyl groups and these interactions seem to prevail over the site-specific adsorption [52]. During these intermolecular interactions the porphyrin core can be deformed and the symmetry of the TPyP molecule modified because the conformation of this molecule is defined by several degree of freedom (dihedral angle in correlation with the rotation of the pyridyl group about $\mathrm{C}-\mathrm{C}$ bond, inclination angle of the same bond and distortion angle determined by the steric repulsion between hydrogen atoms of the pyridyl group and the pyrrole moieties [53]).

The spectra in Figure 30 have revealed a wide absorption band situated between $400 \mathrm{~nm}$ and $600 \mathrm{~nm}$ in PTCDA with a maximum at $480 \mathrm{~nm}$ and a shoulder at $550 \mathrm{~nm}$, this shape being determined by the interactions of the $\pi$-electrons system of the neighbour planar molecules very closed packed in solid state $[21 ; 54]$. The excited states can be the result of the superposition of the intramolecular Frenkel excitons and intermolecular charge transfer excitons existing near the excitation threshold [55]. The UV-VIS spectra of Alq3 confirm that the low temperature isomer (median) correlated with the presence of the weak absorption band situated at $380 \mathrm{~nm}$, dominates in the films deposited at room temperature [56]. For $\mathrm{ZnPc}$ we have evidenced two absorption peaks situated at $690 \mathrm{~nm}$, a strong band corresponding to $\mathrm{Q}$ band and a weak band situated at $330 \mathrm{~nm}$ corresponding to $\mathrm{B}$ band [57]. 
Modifications in the deposition parameters (target concentration, fluence and number of pulses) are reflected in the thickness of the layer and not in the shape of the transmission.

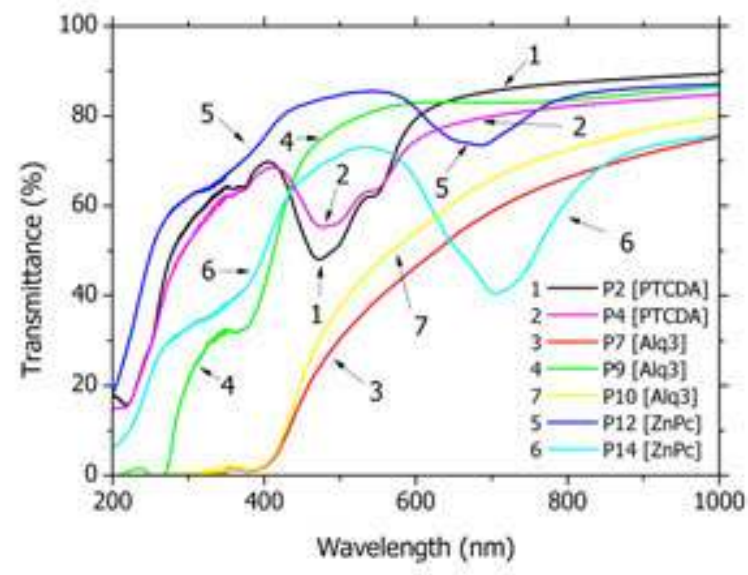

Figure 30. UV-VIS spectra of organic thin films deposited by MAPLE: PTCDA on quartz (1 and 2); PTCDA on ITO (7); Alq3 on quartz (3 and 4); ZnPc on quartz (5 and 6) [54].

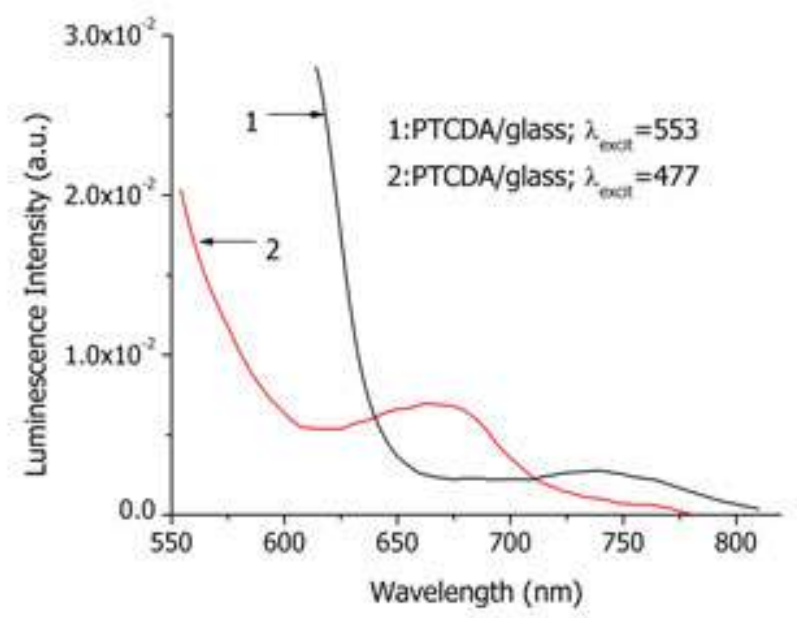

Figure 31. Emission spectra of PTCDA film deposited by vacuum evaporation on glass for two excitation wavelengths [40]. 


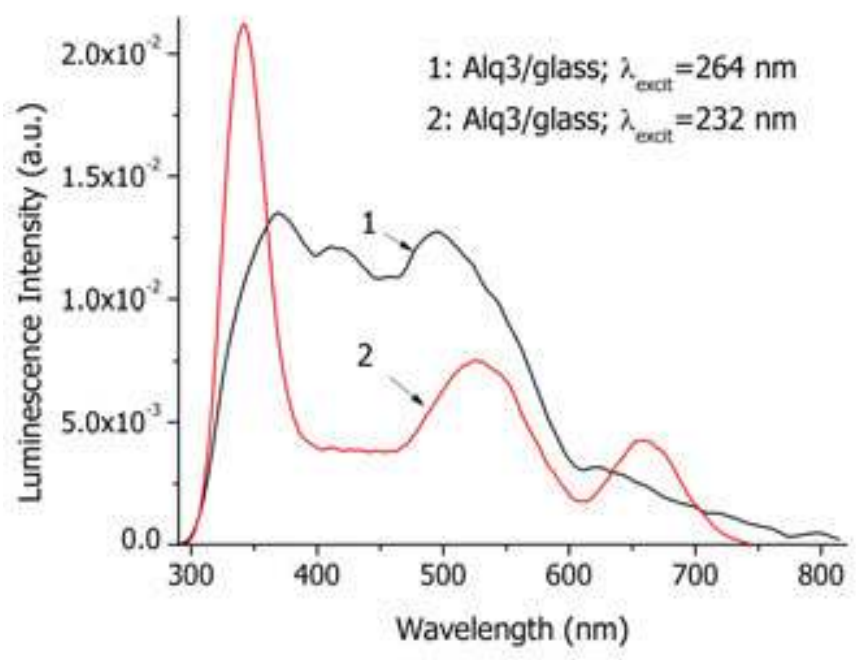

Figure 32. Emission spectra of Alq3 film deposited by vacuum evaporation on glass for two excitation wavelength [40].

The fluorescence emission spectrum of PTCDA film presented in Figure 31 shows a broad structureless band shifted significantly to the red compared to the PTCDA fluorescence spectrum in solution as a consequence of a strong interaction between the organic molecules in the solid state favoured by the close spacing and important overlap of the molecular planes. The emission takes place in PTCDA from the lowest excited singlet state $\left(\mathrm{S}_{1}\right)$ by the relaxation of the electron transferred by the light absorption on an antibonding $\pi$ orbital [40]. The preservation of the emission peak situated at $\lambda=680 \mathrm{~nm}$ for two different excitation wavelengths sustains the compositional homogeneity of the film. The luminescence in Alq3, presented in Figure 32, is generated by excitations localized on individual molecule with optical properties independent of molecular environment [58]. The presence of two isomers with different spatial configurations is sustained by the generation of different emission peaks for different excitation wavelengths. The significant Stocks shift in the both spectra of PTCDA $(\triangle \mathrm{E}=0.40 \mathrm{eV})$ and Alq3 $(\Delta \mathrm{E}=0.9 \mathrm{eV})$ between the peaks of the lowest level absorption and highest fluorescence emission level, and large Frank-Condon shift (0.40-2.3 eV) measured peak to peak between the absorption and emission spectra, can be correlated with effects determined by the solid state structure and with important conformational differences between the ground state and the excited state [40]. 


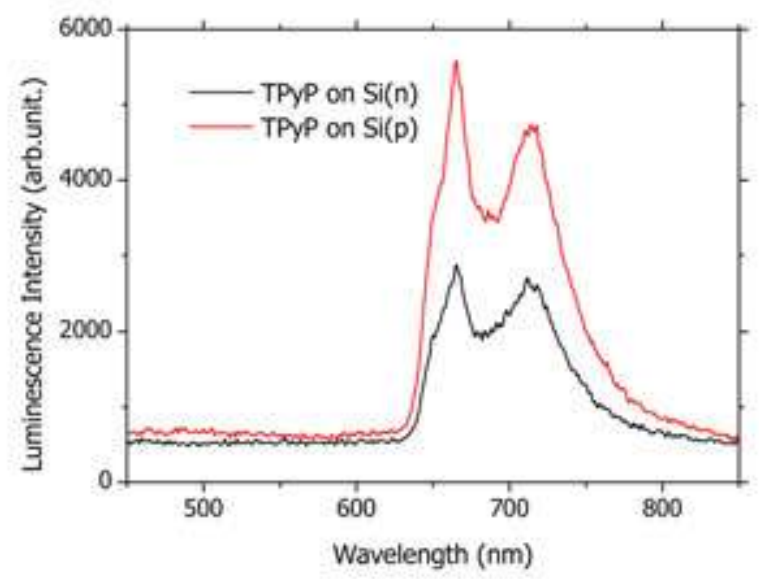

Figure 33. Photoluminescence spectra of TPyP films deposited by vacuum evaporation on different types of Si substrates [46].

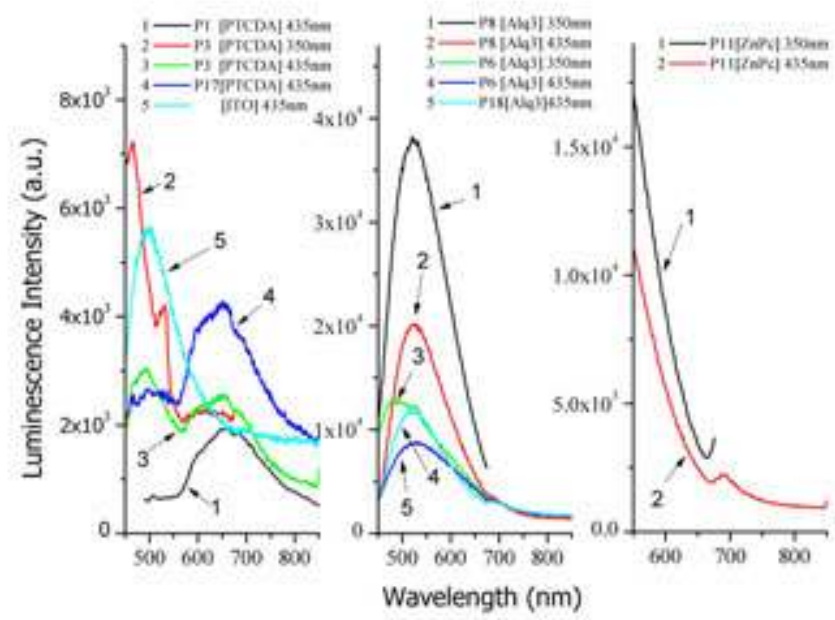

Figure 34. Photoluminescence spectra of organic thin films deposited by MAPLE for two excitation wavelengths: (a) PTCDA on Si (1 and 2), PTCDA on ITO/ZnPc (4); (b) Alq3 on Si (1 and 3), Alq3 on ITO/ZnPc (5); (c) ZnPc on Si (1) [54].

The photoluminescence spectra of TPyP films deposited on Si by vacuum evaporation have revealed in Figure 33 two emission bands situated at $660 \mathrm{~nm}$ and $700 \mathrm{~nm}$ associated to $\mathrm{Q}$ bands and corresponding to free-base ethio type porphyrin [46]. 
The photoluminescence investigations have evidenced the preservation of the chemical structure of the compounds (PTCDA, Alq3, ZnPc) during the deposition by MAPLE, because we have identified the characteristic emission peaks corresponding to each compound, as can be seen in Figure 34. The emission peak situated at $500 \mathrm{~nm}$, in PTCDA deposited on $\mathrm{Si}$, is associated with monomer-like species and that situated at $650 \mathrm{~nm}$ can be associated with two excimeric states [54]. The emission peak in Alq3 film deposited on Si is situated at $520 \mathrm{~nm}$ being associated with the excitation of median isomer dominant at the deposition temperature. The emission peak in $\mathrm{ZnPc}$ film deposited on $\mathrm{Si}$ is situated between $650 \mathrm{~nm}$ and $750 \mathrm{~nm}$ and can be associated with the deexcitation from the first excited singlet state with an energy of $1.8 \mathrm{eV}$ [54]. In the heterostructures with double organic layer the weak emission of $\mathrm{ZnPc}$ is masked by the stronger emission of PTCDA and Alq3.

\subsection{Electrical properties of aromatic derivatives thin films}

A material could become interesting for optoelectronic application if it is adequate from the point of view of both optical and electrical properties (such as good contact inject and transport the charge carriers). A good injection of the charge carrier was evidenced at the ITO/mDNB contact compared to ITO/benzil contact as can be shown in Figure 35. This can be explain by the difference in the energetic contact barrier, which is higher between ITO and benzil than ITO and m-DNB, as a consequence of the position of the highest occupied molecular orbital (HOMO) and lowest unoccupied molecular orbital (LUMO) in these aromatic derivative compounds.

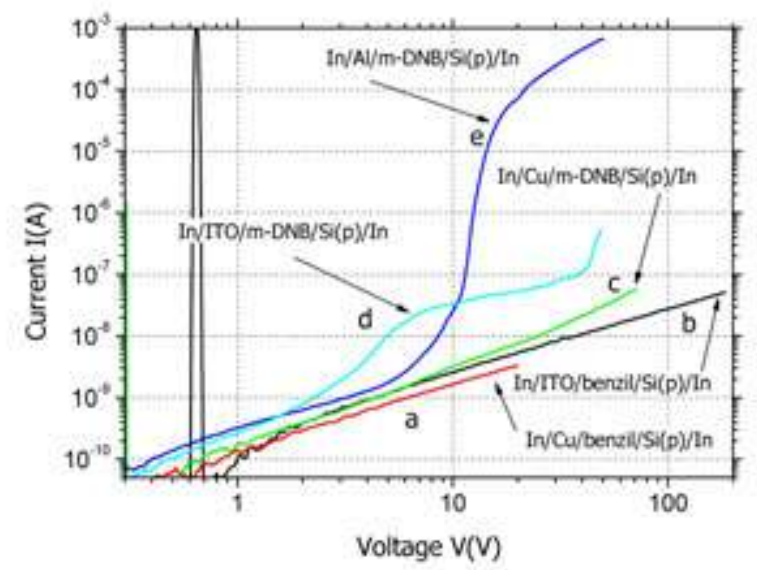

Figure 35. I-V characteristics of the benzil and $\mathrm{m}$-DNB based heterostructures with $\mathrm{Si}(\mathrm{p})$ anode and different cathode (Cu, ITO, Al): (a) Cu for benzil ; (b) ITO for benzil; (c) Cu for m-DNB; (d) ITO for m-DNB, (e) Al for m-DNB [61]. For contacting we have used very high purity In. 
For the sample based on m-DNB two different regions were identified on the I-V characteristic: the ohmic behaviour region at low voltages and a region with a behaviour associated to the space charge limited effect at voltages $>5 \mathrm{~V}$. Both in Figure 35 and Figure 36 can be seen that at voltages $>5 \mathrm{~V}$ the effect of the space charge limitation of the current becomes important in the heterostructure ITO/m-DNB/Si(p). The steep increase in the current at voltage $\sim 10 \mathrm{~V}$ for $\mathrm{Al} / \mathrm{m}-\mathrm{DNB} / \mathrm{Si}(\mathrm{p})$ and ITO/m-DNB/Si(p) can be associated with an avalanche generation mechanism involving energetic states situated in the band gap, in the interface region, state generated by the easy diffusion of $\mathrm{Al}$ in organic layer favoured by the first ionization potential of $\mathrm{Al}(5.98 \mathrm{eV})$.

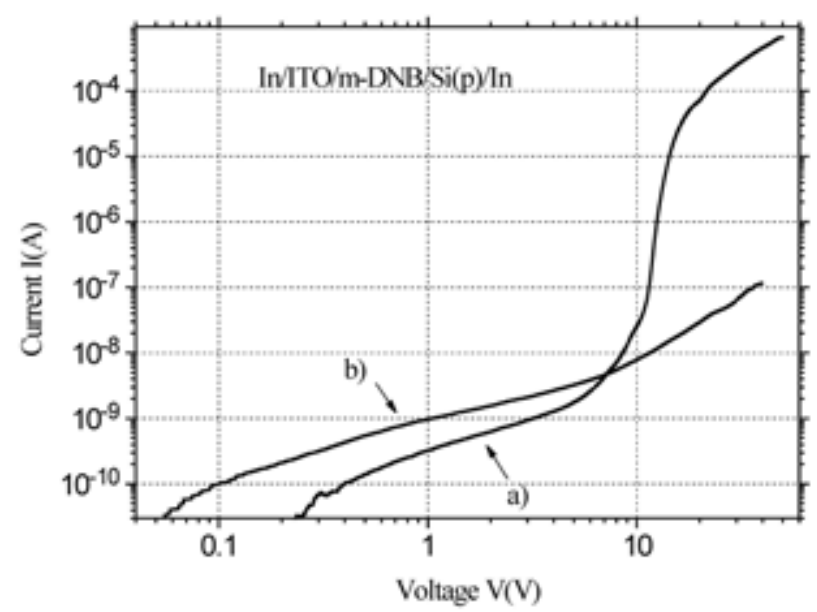

Figure 36. I-V characteristics for ITO/m-DNB/Si(p) heterostructures for different type ITO contacts. For contacting we have used very high purity In [61].

The different ITO/m-DNB/Si heterostructures have shown significantly different shapes of the I-V characteristics as a consequence of the crystalline quality of the organic layer, in correlation with the preparation method (rapid thermal directional solidification).

A blocking diode behaviour, both at direct and reverse bias, has been emphasized in Figure 37 at low applied voltages in ITO/TPyP/Si heterostructures, independent of the type of conduction of the Si electrode. For these heterostructures no photoelectric effect has been evidenced. These I-V characteristics are quasi-linear at low voltages and at higher voltages the limitation of the current determined by the space charge and/or by trap-charge became very important as can be seen in Figure 37. 

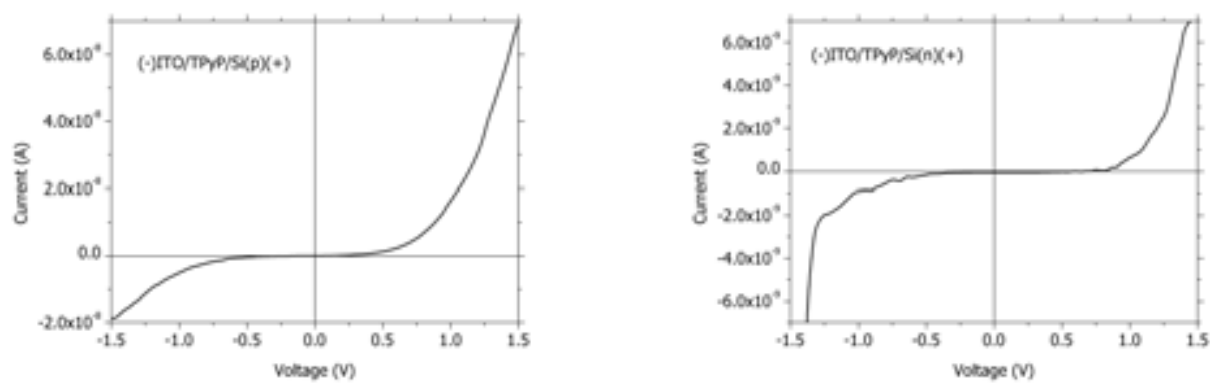

Figure 37. I-V characteristics for ITO/TPyP/Si heterostructures [46].

The heterostructure $\mathrm{Au} / \mathrm{TPyP} / \mathrm{Si}$, at an illumination through the metallic electrode and direct polarization, shows a rectifier behaviour presented in Figure 38, determined by the energetic barrier at the contact $\mathrm{Au} / \mathrm{TPyP}$, which can be lowered applying a voltage $>0.30 \mathrm{eV}$. The linear behaviour at low applied voltages became a power dependence with $n>2$ at voltages $>0.1$ $\mathrm{V}$ and corresponds to trap charge limited current. At reverse bias, the same heterostructure shows a blocking behaviour independent of the applied voltage, because the energetic barrier is too high and can't be surpass by the charge carriers [46].

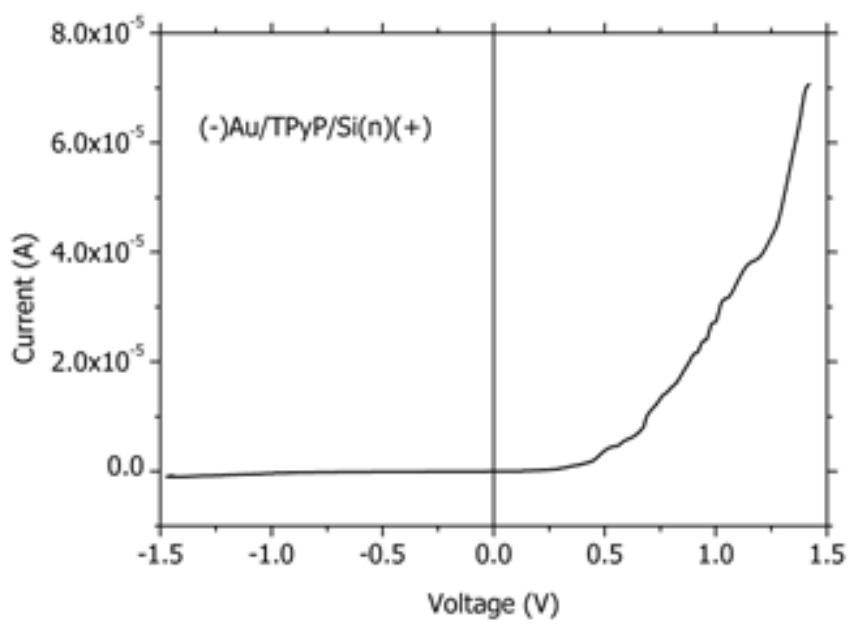

Figure 38. I-V characteristic of the Au/TPyP/Si heterostructure [46]. 

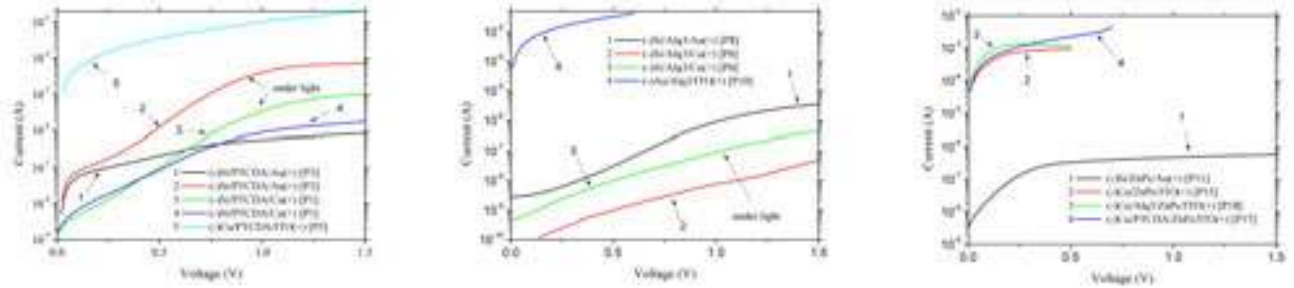

Figure 39. I-V characteristics of Si or ITO/organic layer(s)/Au or Cu heterostructures prepared by MAPLE: based on PTCDA (a) curves 2 and 3 under illumination; based on Alq3 (b) curve 3 under illumination; based on ZnPc (c) all in dark [54].

In Figure 39 a, b, c are presented the I-V characteristics in dark and under illumination, the highest current $\left(\sim 10^{-4}-10^{-3} \mathrm{~A}\right)$ being obtained in dark with the structure prepared with PTCDA, Alq3 or ZnPc on ITO substrate, at low applied voltage of $0.5 \mathrm{~V}$ [54]. This current is with three orders higher than the current for the same structure realized on Si. This behaviour is correlated, in the first case, with the height of the energetic barriers at the interfaces that favour the injection of holes from ITO positively biased in organic. The I-V characteristics obtained under continuous illumination at an applied voltage of $1 \mathrm{~V}$, indicate a higher current in the heterostructures realized with PTCDA and, Si and Cu electrodes, explained by the higher energetic barrier for electron injection at the contact $A l q 3 / \mathrm{Cu}(\Delta \mathrm{E}=1.5 \mathrm{eV}) \mathrm{com}$ pared to PTCDA $/ \mathrm{Cu}(\triangle \mathrm{E}=0.7 \mathrm{eV})$. The current is one order higher than the dark current confirming the photo generation process [54]. In the heterostructures with double organic layer and, ITO and $\mathrm{Cu}$ electrodes, we have obtained a current of $2 \times 10^{-3} \mathrm{~A}$ at $0.5 \mathrm{~V}$, explained by the energetic barrier in $\mathrm{ITO} / \mathrm{ZnPc} / \mathrm{Alq} 3 / \mathrm{Cu}$ heterostructure and by the presence of the interface dipoles reducing the energetic barrier and improving the conduction in ITO/ZnPc/ PTCDA/Cu heterostructure.

\section{Organic/organic composite films based on aromatic derivative inclusions for optoelectronic applications}

Lately, a special attention has been paid to composite materials based on different organic polymeric matrix and organic inclusions to obtain materials combining the properties of the both components $[25 ; 34 ; 45 ; 62 ; 63]$. This field of research has developed from fundamental investigations to the synthesis of new monomers to be introduced in polymeric matrix. The most important advantage of the polymeric matrix is the possibility to deposit thin films using inexpensive methods, such as the deposition from solution by spin-coating. The limiting parameter is the quality of the layer and can be controlled by the control of the experimental conditions. A special attention is focused to the identification and development of $\pi$-conjugated systems with functional groups that assure an improvement in the emission properties and charge carrier mobility necessary for optoelectronic applications. 
We have emphasised the effect of the polycarbonate of bisphenol A matrix on the properties of the synthesised amidic monomers with $-\mathrm{CN}$ and $-\mathrm{NO}_{2}$ substituent groups with the purpose to manipulate the local molecular environment of the monomer for changing the physical properties of the films (transmission, luminescence, electrical transport) in correlation with the quality of the spin-coated layers.

The polycarbonate of bisphenol A, utilized as matrix, is characterised by a large domain of transparency, high transmission in visible, high refraction index, solubility in common solvents. As inclusions, to be embedded in the matrix, we have selected monomers characterised by a maleamic acid structure with different functional groups:

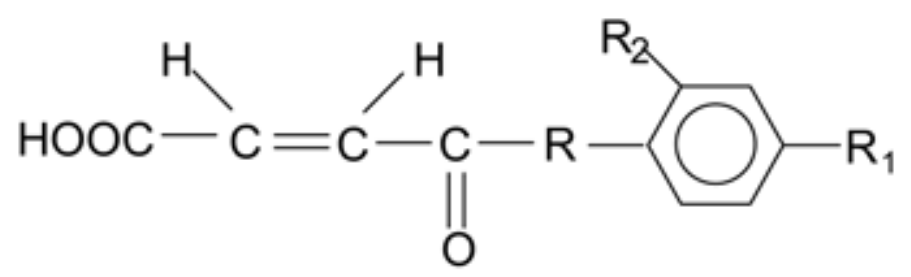

where $\mathrm{R}=-\mathrm{NH}, \mathrm{R}_{1}=-\mathrm{CN}$ for $(\mathrm{MM} 3) ; \mathrm{R}=-\mathrm{NH}, \mathrm{R}_{1}=-\mathrm{NO}_{2} ; \mathrm{R}_{2}=-\mathrm{NO}_{2}$ for (MM5) [64].

After testing the process of layer formation in correlation with the surface energy by contact angle measurements using two different solvents, we have selected dimethylformamide (DMF) for the preparation of the "mother solution" that contain the both components, matrix and inclusion. We have varied the weight ratio between the components $1 / 3 ; 1 / 2 ; 1 / 1$, using the pre-wetting of the surface and different duration and rotation speeds for the spreading stage ( $\left.\mathrm{t}_{1}=3 \mathrm{~s} ; 6 \mathrm{~s} ; 9 \mathrm{~s} ; 12 \mathrm{~s} ; \mathrm{v}_{1}=0.5 \mathrm{krpm} ; 0.7 \mathrm{krpm} ; 0.9 \mathrm{krpm} ; 1.13 \mathrm{krpm}\right)$ and homogenisation stages $\left(\mathrm{t}_{2}=10 \mathrm{~s} ; 20 \mathrm{~s} ; \mathrm{v}_{2}=1.6 \mathrm{krpm} ; 1.9 \mathrm{krpm} ; 2.2 \mathrm{krpm} ; 2.7 \mathrm{krpm} ; 3 \mathrm{krpm}\right)$, with the purpose to identify the most adequate conditions for the deposition of layers [64].

UV-Vis transmission spectra presented in Figure 40 have evidenced differences in the behaviour of the composite material prepared with (MM3) and (MM5), determined by differences in the chemical structure of these components. The shape of the transmission curve is determined by the substituent to the aromatic nucleus and depends on the lone electron pairs of the oxygen atoms in the carbonyl and nitrous groups involving $\left(n, \pi^{*}\right)$ state, which are splitted because of the interaction in the solid state between the polycarbonate matrix and (MM5) monomer. No significant difference has been emphasised, in the UV-VIS spectra, between the monomer deposited by vacuum evaporation and the same monomer embedded in a polymeric matrix and deposited by spin-coating. Although (MM3) shows also lone electron pairs the interaction between the cyan groups and the carbonyl groups is not so intense in the solid state to favour the splitting of the $\left(n, \pi^{*}\right)$ energetic level. 
a)

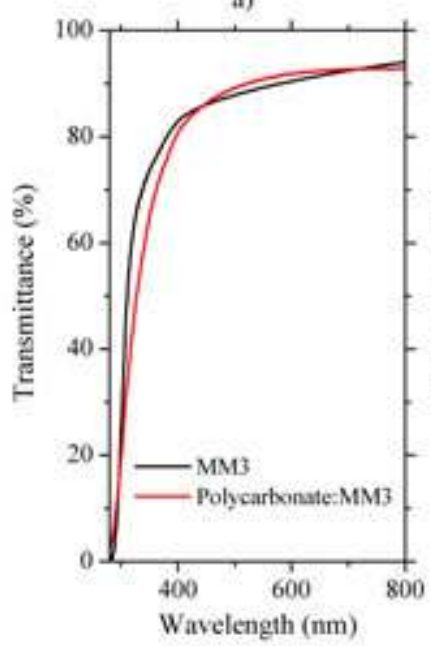

b)

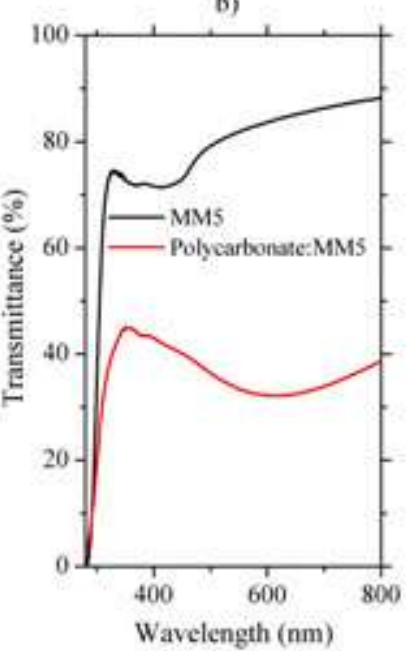

Figure 40. Comparative UV-VIS spectra of MM3 and MM5 monomers deposited by vacuum evaporation and polycarbonate /MM3 and polycarbonate /MM5 deposited by spin coating, on glass substrate [64].

a)

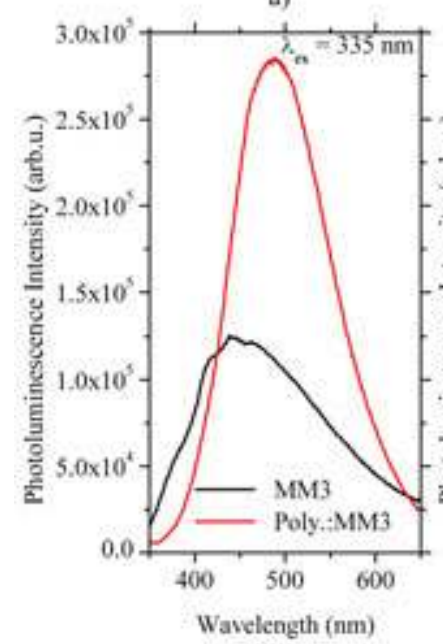

b)

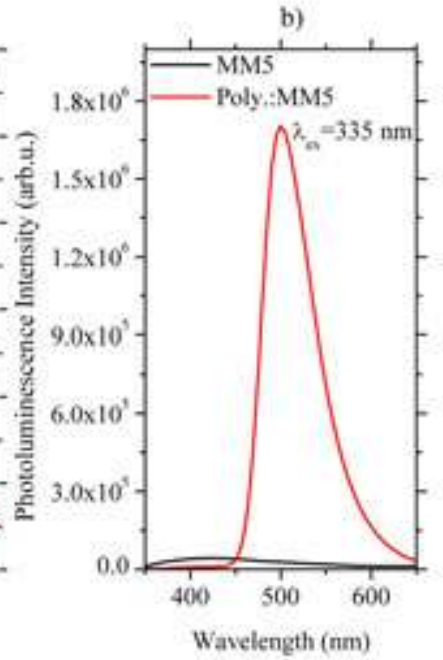

Figure 41. Photoluminescence spectra of monomers (MM3) and (MM5) deposited by vacuum evaporation and polycarbonate/MM3 and polycarbonate/MM5 deposited by spin coating on glass substrates [64]. 
The emission of the polymer/monomer composite material is determined by the interaction between the chromophoric groups in monomer with the polymeric matrix. This interaction can generate the shift, broadening or strengthening of the emission peak [64].

The Figure 41 shows that the polymeric matrix significantly affects the emission spectra of the monomers characterised by a peak situated at $430 \mathrm{~nm}$ in (MM5) and $450 \mathrm{~nm}$ in (MM3). These shapes of the spectra can be correlated with the emission properties of the substituted benzene nucleus [65] and with the involvement of the (n, $\left.\pi^{*}\right)$ states lower than the usual singlet excited states. The strongest emission was obtained for monomer (MM5) in polycarbonate of bisphenol A matrix probably due to the strong absorption of the excitation radiation $(\lambda=335 \mathrm{~nm})$ assuring a higher efficiency of the emission process.

In composite material based on (MM5) the emission spectra show a maximum around 510 $\mathrm{nm}$ and in composite material based on (MM3) a slightly weaker and broader maximum around $480 \mathrm{~nm}$. Figure 41 has not evidenced a strong broadening effect of the matrix on the emission spectrum of monomers. The emission of polycarbonate:MM5 is not blue shifted and therefore we suppose that the monomer is not highly stressed in the polycarbonate matrix.

In Figure 42 are presented some results on the investigation of the effect of the polymeric matrix on the electrical transport properties of Si/monomer/Si and Si/polycarbonate:monomer/Si heterostructures.
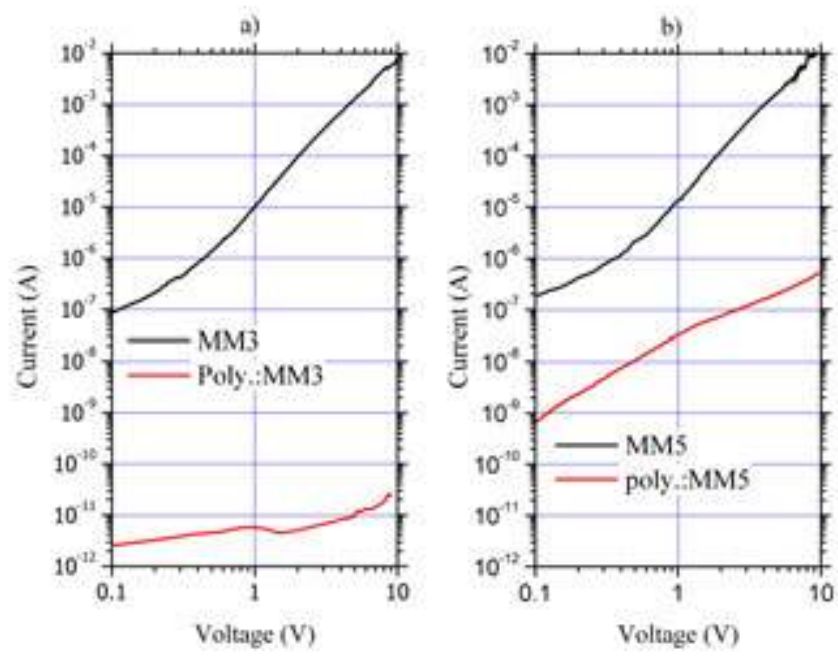

Figure 42. I-V characteristics of Si/MM3/Si and Si/polycarbonate:MM3/Si heterostructures (a) and Si/MM5/Si and Si/ polycarbonate/MM5/Si heterostructures (b) [64].

We have analysed the electrical properties of the heterostructure $\mathrm{Si} / \mathrm{monomer} / \mathrm{Si}$ and $\mathrm{Si} /$ polycarbonate:monomer/Si at room temperature, testing the reproducibility of the measure- 
ments and considering only the typical behaviour. The heterostructures realized with composite materials show a current with 3 orders lower than the heterostructures realized only with monomers. The charge carrier transport is mainly affected by the insulating character of the polymeric matrix. The highest current $\left(3 \times 10^{-8} \mathrm{~A}\right)$ has been obtained in heterostructure Si/polycarbonate:MM5/Si for an applied voltage of $1 \mathrm{~V}$ and for voltages between $0.1 \mathrm{~V}$ and 1 $\mathrm{V}$ the characteristic is weakly superlinear [61]. For the heterostructures realized only with monomers the I-V characteristics are linear at low voltages and become strongly superlinear for voltages $>0.2 \mathrm{~V}$.

The films obtained from the polycarbonate containing the monomer with two nitrous substituents (MM5) to the aromatic nucleus have shown good transparency, and photoluminescence in the green region and promising electrical properties at voltages $>0.6 \mathrm{~V}\left(\mathrm{I}=10^{-8} \mathrm{~A}\right)$ with a close to linear characteristic at voltages between $1 \mathrm{~V}$ and $10 \mathrm{~V}$ [64].

Also this organic/organic composite material seems to be promising for optoelectronic applications, the spin coated composite layers are characterised by a specific morphology and a high degree of disorder which affect the optical and electrical properties and make difficult their control.

\section{Conclusions}

In this chapter we summarize some of the most important results of our work in the field of new materials for applications in the field of optoelectronics. Our interest was focused on organic molecules containing electrons, occupying non-localised molecular orbitals in strongly conjugated systems, such as aromatic derivatives compounds (benzil, m-DNB, PTCDA, ZnPc, Alq3, TPyP) for which we have evidenced large transparency domain and good fluorescence emission.

We have realised a comparative investigation on the properties of the same aromatic derivative compound as bulk and thin film material showing both good optical, including luminescent, properties. The interest in studying bulk organic crystals is justified by the perspective to use these materials as a crystalline host matrix both for organic and inorganic guests/inclusions. The organic matrix assures an efficient fluorescence mechanism and from the guest component it is expected an improvement in stability, emission properties of the matrix and electrical mobility. A special attention was paid to the preparation methods both for bulk crystals (emphasising the correlation between the growth interface stability and quality of the organic crystal) and thin films (emphasising the effect of the thin film deposition method -directional solidification, vacuum evaporation, MAPLE- on the properties of the organic film and heterostructures).

Thin films from the above mentioned aromatic derivatives are preferred as organic matrix for host/guest systems because of the major problems associated with bulk crystalline matrices determined by the difficulties of their growing, processing and doping to assure homogeneous distribution of the guest atoms. 
Important results have been bought in the field of molecular organic matrix based on aromatic derivatives (benzil, m-DNB)/inorganic (iodine, silver, sodium) or/and organic (mDNB, naphthalene, bulk) composite systems and in the field of composite films prepared from polymeric matrix and active monomeric inclusions based on $\pi$-conjugated systems containing functional groups with special properties, to improve the properties of film forming, emission properties of the matrix and the charge carrier mobility in the matrix, with the purpose to obtain materials for potential optoelectronic applications.

We have emphasised the effect of the polycarbonate of bisphenol A matrix on the properties of the synthesised amidic monomers with $-\mathrm{CN}$ and $-\mathrm{NO}_{2}$ substituent groups with the purpose to modify the local molecular environment of the monomer and change the optical and electrical properties of the films.

\section{Author details}

Florin Stanculescu ${ }^{1^{*}}$ and Anca Stanculescu ${ }^{2}$

*Address all correspondence to: fstanculescu@fpce1.fizica.unibuc.ro

1 University of Bucharest, Bucharest-Magurele,, Romania

2 National Institute of Materials Physics, Bucharest-Magurele,, Romania

\section{References}

[1] Helfrich, W., \& Schneider, W. G. (1965). Recombination radiation in anthracene crystals. Phys Rev. Lett., 14(7), 229-231.

[2] Tang, C. W., \& Van Slyke, S. A. (1987). Organic electroluminescent diodes. Appl. Phys. Lett. , 51(12), 913-915.

[3] Van Slyke, S. A., Chen, C. H, \& Tang, C. W. (1996). Organic electroluminescent devices with improved stability. Appl. Phys. Lett., 69(15), 2162-2160.

[4] Kijima, Y., Asai, N., \& Tamura, S. I. (1999). A blue organic light emitting diode. Jpn. J. Appl. Phys.Part 1 , 38(9A), 5274-5277.

[5] Hung, L. S., Tang, C. W., \& Mason, M. G. (1997). Enhanced electron injection in organic electroluminescence devices using an Al/LiF electrode. Appl. Phys. Lett. , 70(2), 152-154.

[6] Parthasarathy, G., Shen, C., Kahn, A., \& Forrest, S. R. (2001). Lithium doping of semiconducting organic charge transport materials. J. Appl. Phys., 89(9), 4986-4992. 
[7] Brütting, W., Riel, H., Beierlein, T., \& Riess, W. (2001). Influence of trapped and interfacial charges in organic multilayer light-emitting devices. J. Appl. Phys. , 89(3), 1704-1712.

[8] Xue, J, \& Forrest, S. R. (2004). Carrier transport in multilayer organic photodetectors: I. Effects of layer structure on dark current and photoresponse. J. Appl. Phys., 95(4), 1859-1868.

[9] Huang, J., Blochwitz-Nimoth, J., Pfeiffer, J. M., \& Leo, K. (2003). Influence of the thickness and doping of the emission layer on the performance of organic light-emitting diodes with PiN structure. J. Appl. Phys. , 93(2), 838-844.

[10] Parthasarathy, G, Burrows, P. E, Khalfin, V, Kozlov, V. G, \& Forrest, S. R. (1998). A metal-free cathode for organic semiconductor devices. Appl. Phys. Lett., 72(17), 2138-2140.

[11] Gu, G., Parthasarathy, G., \& Forrest, S. R. (1999). A metal-free, full-color stacked organic light-emitting device. Appl. Phys. Lett. , 74(2), 305-307.

[12] Ichikawa, M., Amagai, J., Horiba, Y., \& Koyama, T. (2003). Dynamic turn-on behavior of organic light-emitting devices with different work function cathode metals under fast pulse excitation. J. Appl. Phys. , 94(12), 7796-7800.

[13] Guo, T. F., Yang, F. S., Tsai, Z. J., \& Feng, G. W. (2006). High-brighness top-emissive polymer light-emitting diodes utilizing organic oxide/Al/Ag composite cathode. Appl. Phys. Lett. 051103-1-051103-3 , 89(5)

[14] Barth, S., Müller, P., Riel, H., Seidler, P. F., Rieß, Vestweber. H., \& Bässler, H. (2001). Electron mobility in tris(8-hydroxy-quinoline)aluminium thin films determined via transient electroluminescence from single- and multilayer organic light-emitting diodes. J. Appl. Phys. , 89(7), 3711-3719.

[15] Kalinowski, J, Picciolo, L. C, Murata, H, \& Kafafi, Z. H. 2001. Effect of emitter disorder on the recombination zone and the quantum yield of organic electroluminescent diodes. J. Appl. Phys., 89(3), 1866-1874.

[16] Sun, J. X., Zhu, X. L., Meng, Z. G., Yu, X. M., Wong, M., \& Kwok, H. S. (2006). An efficient stacked OLED with double-sided light emission SID International Symposium. Digest of Technical Papers SID Symposium Digest, 37, 1193-1196.

[17] Shao, Y., \& Yang, Y. (2005). Organic solid solutions: formation and applications in organic light-emitting diodes. Adv. Funct. Mater, 15(12), 1781-1786.

[18] Burroughes, J. H., Bradley, D. C., Brown, A. R., Marks, R. N., Mackay, K., Friend, R. H., Burn, P. L., \& Holmes, A. B. (1990). Light-emitting diodes based on conjugated polymers. Nature, 347(6293), 539-541.

[19] Tse, S. C., Tsung, K. K., \& So, S. K. (2007). Single-layer organic light-emitting diodes using naphthyl diamine. Appl. Phys. Lett. , 90(21), 213502. 
[20] Lu, M. H., \& Sturm, J. C. (2002). Optimization of external coupling and light emission in organic light-emitting devices: modeling and experiment. J. Appl. Phys. , 91(2), 595-604.

[21] Stanculescu, A., Antohe, S., Alexandru, H. V., Tugulea, L., Stanculescu, F., \& Socol, M. (2004). Effect of dopant on the intrinsic properties of some multifunctional aromatic compounds films for target applications. Synthetic Metals, 147(1-3), 215-220.

[22] Stanculescu, A., \& Popina, Al. (1996). M -Dinitrobenzene optical nonlinear organic crystals growth for optoelectronics. SPIE Proc, 2700.

[23] Stanculescu, A., Stanculescu, F., \& Alexandru, H. (1999). Melt growth and characterization of pure and doped meta-dinitrobenzene crystals. J. Cryst. Growth, 198/199(1-4), $572-577$.

[24] Stanculescu, A. (2007). Investigation of the growth process of organic/inorganic doped aromatic derivatives crystals. J. Optoelectron. Adv. Mater. , 9(5), 1329-1336.

[25] Stanculescu, A., Tugulea, L., Alexandru, H. V., Stanculescu, F., \& Socol, M. (2005). Molecular organic crystalline matrix for hybrid organic-inorganic (nano) composite materials. J. Cryst. Growth, 275(1-2), e1779-e1786.

[26] Sekerka, R. F. (1968). Morphological Stability. J. Cryst. Growth, 3(4), 71-81.

[27] Mullins, W. W., \& Sekerka, R. F. (1963). Morphological Stability of a Particle Growing by Diffusion or Heat Flow. J. Appl. Phys. , 34(2), 323-329.

[28] Mullins, W. W., \& Sekerka, R. F. (1964). Stability of a Planar Interface During Solidification of a Dilute Binary Alloy. J. Appl. Phys. , 35(2), 444-451.

[29] Flemings, M. C. (1974). Solidification processing. Mc.Graw-Hill, Inc.

[30] Kitaigorodski, A. I. (1966). J. Chem. Phys. Phys. Chem Biol., 63, 9-16.

[31] Kitaigorodski, A. I. (1973). Molecular crystals and molecules. Academic Press, New York.

[32] Halfpenny, P. J., Ristic, R. I., Shepherd, E. E. A., \& Sherwood, J. N. (1993). The crystal growth behaviour of the organic non-linear optical material 2-( $\alpha$-methylbenzylamino)-5nitropyridine (MBANP). J. Cryst. Growth, 128(1-4), 970-975.

[33] Leonard, N. J., \& Blout, E. R. (1950). The Ultraviolet Absorption Spectra of Hindered Benzils. J. Am. Chem. Soc. , 72(484), 487.

[34] Stanculescu, A., Mihut, L., Stanculescu, F., \& Alexandru, H. (2008). Investigation of emission properties of doped aromatic derivative organic semiconductor crystals. J. Cryst. Growth, 310(7-9), 1765-1771.

[35] Windsor, M. W. (1965). Luminescence and energy transfer in: D. Fox, M. M. Labes, A. Weissberger (eds.). Physics and chemistry of the organic solid state, vol. II, Interscience, New York, 343. 
[36] Rothe, C., Guentner, R., Scherf, U., \& Monkman, A. P. (2001). Trap influenced properties of the delayed luminescence in thin solid films of the conjugated polymer poly(9,9- di(ethylhexyl)fluorene). J. Chem. Phys. , 115(20), 9557-9562.

[37] Bardeen, J., \& Brattain, W. H. (1948). The transistor, a semi-conductor triode. Phys. Rev. , 74(2), 230-231.

[38] Schockley, W. (1949). The theory of p-n junctions in semiconductors and p-n junction transistors. Bell System Technical Journal, 28(3), 435-489.

[39] Gaffo, L., Cordeiro, M. R., Freitas, A. R., Moreira, W. C., Gitotto, E. M., \& Zucolotto, V. (2010). The effects of temperature on the molecular orientation of zinc phthalocyanine films. J. Mater. Sci. , 45(5), 1366-1370.

[40] Stanculescu, A., Stanculescu, F., Tugulea, L., \& Socol, M. (2006). Optical properties of 3,4,9,10-perylenetetracarboxylic dianhydride and 8 -hyfroxyquinoline aluminium salt films prepared by vacuum deposition. Mat. Sci. Forum, 514-516, Part 2, 956-960.

[41] Wu, C. I., Hirose, Y., Sirringhaus, H., \& Kahn, A. (1997). Electron-hole interaction energy in the organic molecular semiconductor PTCDA. Chem. Phys. Lett. , 272(1-2), 43-47.

[42] Zugang, L., \& Nazarè, H. (2000). White organic light-emitting diodes from both hole and electron transport layers. Synthetic Metals, 111-112, 47-51.

[43] Curioni, A., \& Andreoni, W. (2001). Computer simulations for organic light-emitting diodes. IBM J of Research and Development, 45(1), 101-114.

[44] Auwärter, W., Weber-Bargioni, A., Riemann, A., Schiffrin, A., Gröning, O., fasel, R., \& Barth, J. V. (2006). Self-assembly and conformation of Tetra-pyridil-porphyrin molecules on Ag(111). J. Chem. Phys., 124(19), 194708-1-194708-6.

[45] Stanculescu, F., Stanculescu, A., \& Socol, M. (2006). Light absorption in meta-dinitrobenzene and benzyl crystalline films. J. Optoelectron. Adv. Mater. , 8(3), 1053-1056.

[46] Socol, M, Rasoga, O, Stanculescu, F, Girtan, M, \& Stanculescu, A. (2010). Effect of the,orphology on the optical and electrical properties of TPyP thin films deposited by vacuum evaporation. Optoelectronics and Advanced Materials-Rapid Communications, 4(12), 2032-2038.

[47] Socol, G., Mihailescu, I. N., Albu, A. M., Antohe, S., Stanculescu, F., Stanculescu, A., Mihut, L., Preda, N., Socol, M., \& Rasoga, O. (2009). MAPLE prepared polymerc thin films for non-linear optic applications. Appl. Surf. Sci. , 255(10), 5611-5614.

[48] Evans, T. R., \& Leermakers, P. A. (1967). Emission spectra and excited-state geometry of $\alpha$-diketones. J. Am. Chem. Soc. , 89(17), 4380-4382.

[49] Kohler, B. E., \& Loda, R. T. (1981). Spectroscopy of the benzil-bibenzyl mixed crystal system: High resolution optical studies. J. Chem. Phys. , 74(1), 18-24. 
[50] Coppens, P., Ma, B. Q., Gerlits, O., Zhang, Y., \& Kulshrestha, P. (2002). Crystal engineering, solid state spectroscopy and time-resolved diffraction. Cryst. Eng. Commun. , 4(54), 302-309.

[51] George, H., \& Guo, Q. (2008). Self-assembled two-dimensional supramolecular architectures of zinc porphyrin molecules on mica. J. Phys.: Conference series, 100(5), 052077 .

[52] Auwärter, W., Klappenberger, F., Weber-Bargioni, A., Schiffrin, A., Strunskus, T., Wöll, C., Pennec, Y., Riemann, A., \& Barth, J. V. (2007). Conformational adaptation and selective adatom capturing of tetrapyridyl-porphyrin molecules on a copper (111) surface. J. Am. Chem. Soc. , 129(36), 11279-11285.

[53] Klappenberger, F., Weber-Bargioni, A., Auwärter, W., Marschall, M., Schiffrin, A., \& Barth, J. V. (2008). Temperature dependence of conformation, chemical state and metal-direct assembly of tetrapyridyl-porphyrin on Cu (111). J. Chem. Phys. , 129(21), 214702-214702.

[54] Stanculescu, A., Socol, M., Socol, G., Mihailescu, I. N., Girtan, M., \& Stanculescu, F. (2011). Maple prepared organic heterostructures for photovoltaic applications. Apll. Phys. A, 104(3), 921-928.

[55] Sadrai, M., Hadel, L., Sauers, R. R., Husain, S., Krogh-Jespersen, K., Westbrook, J. D., \& Bird, G. R. (1992). Lasing action in a family of perylene derivatives: singlet absorption and emission spectra, triplet absorption and oxygen quenching constants and molecular mechanics and semiempirical molecular orbital calculations. J. Phys. Chem. , 96(20), 7988-7996.

[56] Jian, Z. A., Luo, Y. Z., Chung, J. M., Tang, S. J., Kuo, M. C., Shen, J. L., Chiu, K. C., Yang, C. S., Chou, W. C., Dai, C. F., \& Yeh, J. M. (2007). Effect of isomeric transformation on characteristics of Alq3 amorphous layers prepared by vacuum deposition at various substrate temperature. J. Appl. Phys. 123708-1-123707-6 , 101(12)

[57] Senthilarasu, S., Sathyamoorthy, R., Latitha, S., Subbarayan, A., \& Natarajan, K. (2004). Thermally evaporated ZnPc thin films-band gap dependence on thickness. Sol. Energy Mater. Sol. Cells, 82(1-2), 179-186.

[58] Burrows, P. E., Shen, Z., Mccarty, D. M., Forrest, S. R., Cronin, J. A., \& Thompson, M. E. (1996). Relationship between electroluminescence and current transport in organic heterojunction light-emitting devices. J. Appl. Phys., 79(10), 7991-8006.

[59] Ferguson, A. J., \& Jones, T. S. (2006). Photophysics of PTCDA and me-PTCDI thin films: effects of growth temperature. J. Phys. Chem.B, 110(13), 6891-6898.

[60] Haas, M., Shi-Xia, L., Kahnt, A., Leiggener, C., Guldi, D. M., Hauser, A., \& Decurtins, S. (2007). Photoinduced energy transfer processes within dyads of metallophthalocyanines compactly fused to a Ruthenium (II) polypyridine chromophore. J. Org. Chem., 72(20), 7533-7543. 
[61] Stanculescu, A., \& Stanculescu, F. (2007). Investigation of the properties of indium tin oxide-organic contacts for optoelectronic applications. Thin Solid Films, 515(24), 8733-8737.

[62] Lee, K. J, Oh, J. H, Kim, Y, \& Jang, J. (2006). Fabrication of photoluminescent-dye embedded poly(methyl methacrylate) nanofibers and their fluorescence resonance energy transfer properties. Adv. Mater, 18(17), 2216-2219.

[63] Koratkar, N. A., Suhr, J., Johsi, A., Kane, R., Schadler, L., Ajayan, P. M., \& Bertolucci, S. (2005). Characterizing energy dissipation in single-walled carbon nanotube polycarbonate composite. Appl. Phys. Lett, 87(6), 06312-1-06312-3.

[64] Stanculescu, F., Stanculescu, A., Girtan, M., Socol, M., \& Rasoga, O. (2012). Effect of the morphology on the optical and electrical properties of polycarbonate film doped with aniline derivatives monomers. Synthetic Metals, 161(23-24), 2589-2597.

[65] Dulcic, A., \& Sauteret, C. (1978). The Regularities Observed in the Second Order Hyperpolarizabilities of Variously Disubstituted Benzenes. J. Chem. Phys. , 69(8), 3453-3457. 